\title{
Optimal Rate Control for Delay-Constrained Data Transmission over a Wireless Channel
}

\author{
Murtaza Zafer, Member, IEEE, and Eytan Modiano, Senior Member, IEEE
}

\begin{abstract}
We study energy-efficient transmission of data with deadline constraints over a time-varying channel. Specifically, the system model consists of a wireless transmitter with controllable transmission rate, time-varying and stochastic channel state, and strict delay constraints on the packets in the queue. While the transmitter can control the rate, the transmission power required depends on the chosen rate and the prevailing channel condition. The objective is to obtain a rate control policy that serves the data within the deadline constraints while minimizing the total energy expenditure. Towards this end, we first introduce the canonical problem of transmitting $B$ units of data by deadline $T$ over a Markov fading channel, and obtain the optimal policy for it using continuous-time stochastic control theory. Using a novel cumulative curves methodology and a decomposition approach, we extend the above setup to consider extensions involving variable deadlines on the packets. Finally, utilizing the analysis we present a heuristic policy for the case of arbitrary packet arrivals to the queue with individual deadline constraints, and give illustrative simulation results for its performance.
\end{abstract}

Index Terms-Delay, Energy, Quality of Service, Stochastic Control, Transmission rate, Wireless.

\section{INTRODUCTION}

Modern wireless systems are projected to carry a large volume of data traffic with an increasing emphasis on quality of service. A significant portion of this traffic would have strict delay requirements arising from various end-user applications that involve real-time data communication. For example, in present commercial data networks, services such as video and real-time multimedia streaming, high throughput file transfers and voice-over-IP impose strict delay constraints on data packets, while, in sensor networks time critical sensing applications impose deadline constraints that require that the data collected must be transmitted back to a central processing entity within a specified time interval. By their nature, wireless systems are characterized by scarcity of resources and one of the critical concerns is energy consumption. Minimizing the energy cost has numerous advantages in terms of efficient battery utilization for mobile devices, increased lifetime of sensor and ad-hoc networks, and better utilization of limited energy sources in satellites. Our work in this paper primarily addresses the above two concerns; specifically, the focus is to utilize dynamic control of the transmission rate of a

This work was supported by NSF ITR grant CCR-0325401, by DARPA/AFOSR through the University of Illinois grant no. F49620-02-10325, by NASA Space Communication Project grant number NAG3-2835 and by ONR grant number N000140610064.

M. Zafer (email: mzafer@us.ibm.com) is with IBM T. J. Watson Research center, Hawthorne, NY 10532, USA, and E. Modiano (email: modiano@mit.edu) is with Massachusetts Institute of Technology, Cambridge, MA 02139, USA. wireless device to minimize energy expenditure subject to packet deadline constraints. Since in most scenarios energy spent for transmission constitutes the bulk of the total energy expenditure, the energy cost in this paper will refer to the transmission energy cost.

Most wireless devices are now equipped with channel measurement and rate adaptation capabilities [1]. Channel measurement allows the transmitter-receiver pair to measure the fade state using a pre-determined pilot signal while rate control capability allows the transmitter to adjust the transmission rate over time. Such a control can be achieved in various ways that include adjusting the power level, symbol rate, coding scheme, constellation size and any combination of these approaches; furthermore, in some technologies the receiver can detect these changes directly from the received data without the need for an explicit rate change control information [2]. Also, transmission rate can be adapted very rapidly over millisecond duration time-slots [1], thus providing ample opportunity to utilize rate adaptation to optimize system performance.

To reliably transmit at a certain rate, there is a minimum amount of transmission power required which depends on the transmitter system; however, for most communication schemes the power-rate function has two fundamental aspects which are common assumptions in the literature [4]-[6], [10][12], [16]. First, for a fixed bit-error probability and channel state, the required transmission power is a convex function of the rate as shown in Figure 1(a). This implies, from Jensen's inequality, that transmitting data at low rates over a longer duration (spreading the transmission over time) is more energy efficient as compared to high rate transmissions. Second, the wireless channel is time-varying which shifts the convex power-rate curves as a function of the channel state as shown in Figure 1(b). As good channel conditions require less transmission power, one can exploit this variability over time by adapting the rate in response to the channel conditions. Thus, by adapting the transmission rate intelligently over time energy cost can be significantly reduced.

In this paper, we seek to address the question of dynamic rate adaptation when data packets have strict deadline constraints. Specifically, we first consider a simplified canonical problem where the transmitter queue has $B$ units of data that must be transmitted by a single deadline $T$ over a fading channel modelled as a general Markov process. Using a continuous-time stochastic control formulation we obtain the optimal transmission policy in explicit/closed form. We then consider the case when the packets in the queue have variable deadline constraints. While this problem is complex to solve, a decomposition approach is presented wherein it is 

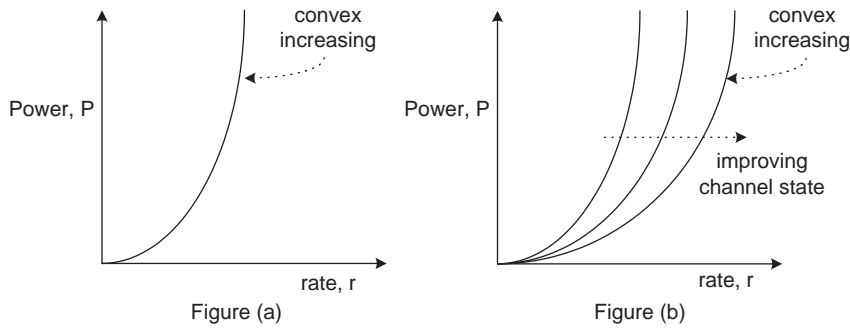

Fig. 1. Transmission power as a function of the rate and the channel state; (a) fixed channel state, (b) variable channel state.

viewed as multiple instances of the earlier canonical problem but with dependencies among them. The rate control policy thus obtained is shown to be optimal under specific instances. Finally, utilizing these results we present a simple heuristic rate control policy for an arbitrary (general) packet arrival process with individual deadline constraints on the data packets, and give illustrative simulation results quantifying the gains in the energy cost achieved by it.

Transmission rate control has been actively studied in the communication networks literature in various different contexts. Adaptive network control and scheduling has been studied in the context of network stability [12], [14], average throughput [13], [15], average delay [4], [16] and packet drop probability [17]. However, this literature considers "average metrics" that are measured over an infinite time horizon and hence do not directly apply for delay constrained/real-time data. Furthermore, adapting the transmission rate simply based on steady state distributions does not suffice and to consider strict deadlines one needs to take into account the system dynamics over time; thus, introducing new challenges and complexity into the problem. Recent work in this direction includes [5]-[8], [10], [11]. The work in [5] studied various offline formulations under complete knowledge of the future and devised heuristic online policies using the offline optimal solutions. The authors in [6] studied several data transmission problems using Dynamic Programming (DP), however, the specific problem that we consider in this work becomes intractable using this methodology due to the large state space in the DP-formulation or the well-known "curse of dimensionality". The works in [10], [11] studied formulations for energy efficient data transmission over a static channel without fading. In our earlier work in [7] we studied energy efficient data transmission over static non-fading channels using a network calculus approach while in [8], [9] we presented specific parts of this work.

\section{SySTEM MODEL}

We consider a continuous-time model of the system. Clearly, such a model is an approximation of the actual system, but the assumption is justified, since in practice transmission rate can be adjusted over time-slots on the order of $1 \mathrm{msec}$ which are much shorter than packet delay requirements usually on the order of 100's of msec [1]. A significant advantage of such a model is that it makes the problem mathematically tractable and yields simple solutions. The alternative discrete-time

\begin{tabular}{|c|c|c|}
\hline modulation & bits/symbol & SNR/symbol \\
\hline 2 PAM & 1 & $0.25 \mathrm{~d}^{2}$ \\
\hline 4 QAM & 2 & $0.50 \mathrm{~d}^{2}$ \\
\hline 16 QAM & 4 & $1.25 \mathrm{~d}^{2}$ \\
\hline 64 QAM & 6 & $5.25 \mathrm{~d}^{2}$ \\
\hline
\end{tabular}

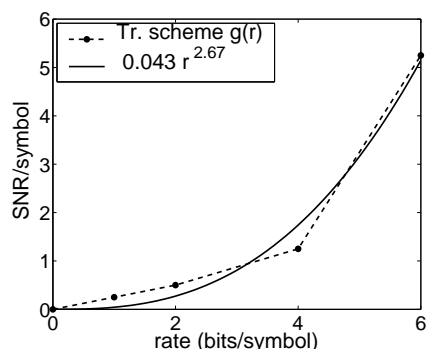

Fig. 2. Modulation scheme considered in [12] as given in the table. The corresponding plot shows the least squares monomial fit, $0.043 r^{2.67}$, to the scaled piecewise linear power-rate curve.

dynamic programming setup is intractable, computationally intensive and would only yield numerical solutions without much insights. Furthermore, the results obtained using the continuous-time model can be applied to the discrete-time system in a very straightforward manner by simply evaluating the solution at discrete times as done for the simulation results in Section V-B.

\section{A. Transmission Model}

Let $h_{t}$ denote the channel gain, $P(t)$ the transmitted signal power and $P^{r c d}(t)$ the received signal power at time $t$. We make the common assumption [4]-[6], [10]-[12], [16] that the required received signal power for reliable communication, with a certain low bit-error probability, is convex in the rate; i.e. $P^{r c d}(t)=g(r(t))$, where $g(r)$ is a non-negative convex increasing function for $r \geq 0$. Since the received signal power is given as $P^{r c d}(t)=\left|\bar{h}_{t}\right|^{2} P(t)$, the required transmission power to achieve rate $r(t)$ is given by,

$$
P(t)=\frac{g(r(t))}{c(t)}
$$

where $c(t) \triangleq\left|h_{t}\right|^{2}$. The quantity $c(t)$ is referred to as the channel state at time $t$. Its value at time $t$ is assumed known either through prediction or direct channel measurement but evolves stochastically in the future. As an example, with optimal channel coding the well-known Shannon capacity formula gives the power per bit as, $P=\frac{N_{0} W\left(2^{r / W}-1\right)}{\left|h_{t}\right|^{2}}$; other examples of (1) can be found in [4], [5]. It is worth emphasizing that while we defined $c(t)$ as $\left|h_{t}\right|^{2}$ to motivate the relationship in (1), more generally, $c(t)$ could include other stochastic variations in the system and (uncontrollable) interference from other transmitter-receiver pairs, as long as the power-rate relationship obeys (1).

In this work, our primary focus will be on $g(r)$ belonging to the class of Monomial functions, namely, $g(r)=k r^{n}, n>$ $1, k>0(n, k \in \mathbb{R})$. While this assumption restricts the generality of the problem, it serves several purposes. First, mathematically it leads to simple optimal solutions in explicitform and insightful observations that can be applied in practice. Second, most importantly, for most practical transmission schemes $g(\cdot)$ is described numerically and its exact analytical form is unknown. In such situations, one can obtain the best approximation of that function to the form $k r^{n}$ by choosing the appropriate $k, n$ and then applying the results thus obtained. 
For example, consider the QAM modulation scheme considered in [12] and reproduced here in Figure 2. The table gives the rate and the normalized signal power per symbol, where $d$ represents the minimum distance between signal points and the scheme is designed for error probabilities less than $10^{-6}$. The plot gives the least squares monomial fit to the transmission scheme and one can see from the plot that for this example the monomial approximation is fairly close. Third, monomials form the first step towards studying extensions to polynomial functions which would then apply to a general $g(\cdot)$ function using the polynomial expansion. Under a more restrictive setting in Section III-C, we also study the class of Exponential functions, namely, $g(r)=k\left(\alpha^{r}-1\right), \alpha>1, k>0(\alpha, k \in \mathbb{R})$. Finally, without loss of generality, throughout the paper we take $k=1$, since any other value of $k$ simply scales the total energy cost without affecting the results on the optimal transmission policy.

\section{B. Channel Model}

We consider a first-order, continuous-time, discrete state space Markov model for the channel state process. Markov processes constitute a large class of stochastic processes that exhaustively model a wide set of fading scenarios and there is substantial literature on these models and their applications [18]-[21]. Denote the channel stochastic process as $C(t)$ and the state space as $\mathcal{C}$. Let $c \in \mathcal{C}$ denote a particular channel state and $\{c(t), t \geq 0\}$ denote a sample path. Starting from state $c$, the channel can transition to a set of new states $(\neq c)$ denoted as $\mathcal{J}_{c}$. Let $\lambda_{c \tilde{c}}$ denote the channel transition rate from state $c$ to $\tilde{c}$, then, the sum transition rate at which the channel jumps out of state $c$ is, $\lambda_{c}=\sum_{\tilde{c} \in \mathcal{J}_{c}} \lambda_{c \tilde{c}}$. Clearly, the expected time that $C(t)$ spends in state $c$ is $1 / \lambda_{c}$ and one can view $\frac{1}{\lambda_{c}}$ as the coherence time of the channel in state $c$.

Now, define $\lambda \triangleq \sup _{c} \lambda_{c}$ and a random variable, $Z(c)$, as,

$$
Z(c) \triangleq \begin{cases}\tilde{c} / c, & \text { with prob. } \lambda_{c \tilde{c}} / \lambda, \tilde{c} \in \mathcal{J}_{c} \\ 1, & \text { with prob. } 1-\lambda_{c} / \lambda\end{cases}
$$

With this definition, we obtain a compact and simple description of the process evolution as follows. Given a channel state $c$, there is an Exponentially distributed time duration with rate $\lambda$ after which the channel state changes. The new state is a random variable which is given as $C=Z(c) c$. Clearly, from (2) the transition rate to state $\tilde{c} \in \mathcal{J}_{c}$ is unchanged at $\lambda_{c \tilde{c}}$, whereas with rate $\lambda-\lambda_{c}$ there are indistinguishable selftransitions. This is a standard Uniformization technique and there is no process generality lost with the new description as it yields a stochastically identical scenario ${ }^{1}$.

Example: Consider a two-state channel model with states $b$ and $g$ denoting the "bad" and the "good" channel conditions respectively. The two states correspond to a two level quantization of the channel gain. If the measured channel gain is

\footnotetext{
${ }^{1}$ Other technical assumptions in the model are as follows. The channel state space, $\mathcal{C}$, is a countable space (it could be infinite), and $\mathcal{C} \subseteq \mathbb{R}^{+}$. The states $c=0, \infty$ are excluded from $\mathcal{C}$ since each of this state leads to a singularity in (1). The set $\mathcal{J}_{c}, \forall c$, is a finite subset of $\mathcal{C}$. Transition rate $\lambda_{c}, \forall c$ is bounded which ensures that $\lambda$ defined as the supremum is finite. For all $c$, the support of $Z(c)$ lies in $\left[z_{l}, z_{h}\right]$, where $0<z_{l} \leq z_{h}<\infty$. This ensures that $C(t)$ does not hit 0 or $\infty$, a.s. (almost surely), over a finite time interval.
}
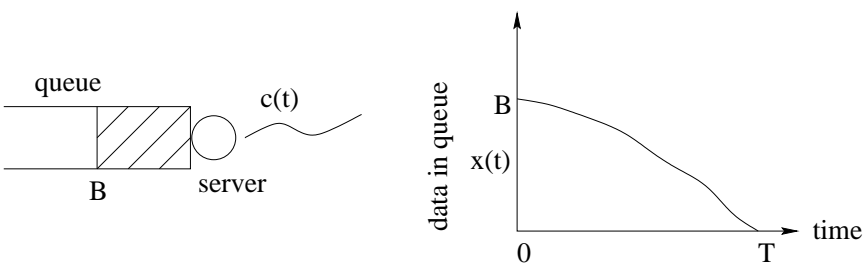

Fig. 3. Schematic description of the system for the $B T$-problem.

below some value, the channel is labelled as "bad" and $c(t)$ is assigned an average value $c_{b}$, otherwise $c(t)=c_{g}$ for the good condition. Let the transition rate from the good to the bad state be $\lambda_{g b}$ and from the bad to the good state be $\lambda_{b g}$. Let $\gamma=c_{b} / c_{g}$, and using the earlier defintion, $\lambda=\max \left(\lambda_{b g}, \lambda_{g b}\right)$. For state $c_{g}$ we have,

$$
Z\left(c_{g}\right)= \begin{cases}\gamma, & \text { with prob. } \lambda_{g b} / \lambda \\ 1, & \text { with prob. } 1-\lambda_{g b} / \lambda\end{cases}
$$

To obtain $Z\left(c_{b}\right)$, replace $\gamma$ with $1 / \gamma$ and $\lambda_{g b}$ with $\lambda_{b g}$ in (3).

\section{BT - PROBLEM}

We begin with the following canonical problem: the transmitter queue has $B$ bits of data and a single deadline $T$ by which this data must be transmitted; the objective is to minimize the expected energy expenditure. We refer to this as the " $B T$-problem" where the notation implies that the amount of data under consideration is $B$, and the deadline is $T$. A more general setup with variable deadline constraints is treated in Section IV. We now describe in detail the control formulation and the optimality conditions for the $B T$-problem.

\section{A. Optimal Control Formulation}

Consider the $B T$-problem and let $x(t)$ denote the amount of data left in the queue at time $t$. The system state can be described as $(x, c, t)$, where this notation means that at the present time $t$, the amount of data left is $x(t)=x$, and the channel state is $c(t)=c$. Let $r(x, c, t)$ denote the chosen transmission rate for the corresponding system state $(x, c, t)$. Since the underlying channel process is Markov, it is sufficient to restrict attention to transmission policies that depend only on the present system state [25]. Clearly then, $(x, c, t)$ is a Markov process. The system is depicted in Figure 3.

Given a policy $r(x, c, t)$, the system evolves in time as a Piecewise-Deterministic-Process (PDP) [23] as follows. It starts with $x(0)=B$ and $c(0)=c_{0}$. Until $\tau_{1}$, where $\tau_{1}$ is the first time instant after $t=0$ at which the channel changes, the queue is reduced at the rate $r\left(x(t), c_{0}, t\right)$. Hence, over the interval $\left[0, \tau_{1}\right], x(t)$ satisfies the ordinary differential equation,

$$
\frac{d x(t)}{d t}=-r\left(x(t), c_{0}, t\right)
$$

Equivalently in integral form, $x(t)=x(0)$ $\int_{0}^{t} r\left(x(s), c_{0}, s\right) d s, t \in\left[0, \tau_{1}\right]$. Then, starting from the new state $\left(x\left(\tau_{1}\right), c\left(\tau_{1}\right), \tau_{1}\right)$, until the next channel transition we have, $\frac{d x(t)}{d t}=-r\left(x(t), c\left(\tau_{1}\right), t\right), t \in\left[\tau_{1}, \tau_{2}\right)$; and this procedure repeats until $t=T$ is reached. A schematic 


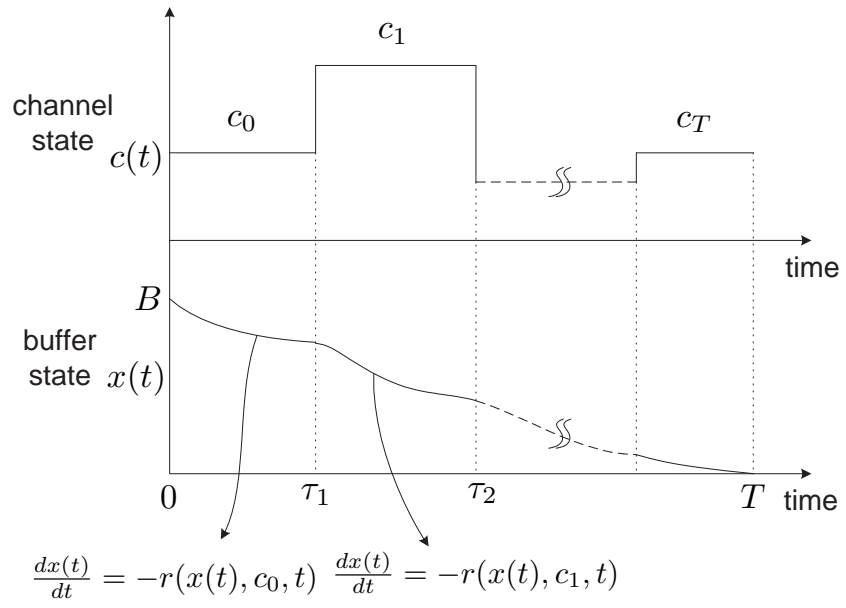

Fig. 4. System evolution over time for the $B T$-problem.

diagram of the process for a particular channel sample path is depicted in Figure 4.

A transmission policy, $r(x, c, t)$, is admissible for the BTproblem, if it satisfies the following,

(a) $0 \leq r(x, c, t)<\infty$, (rate must be non-negative)

(b) $r(x, c, t)=0$, if $x=0$ (no data left to transmit) and,

(c) $x(T)=0$, a.s. (deadline constraint) ${ }^{2}$.

Consider now an admissible transmission policy $r(\cdot)$ and define a cost-to-go function, $J_{r}(x, c, t)$, as the expected energy cost incurred starting from state $(x, c, t), t<T$. Then,

$$
J_{r}(x, c, t)=E\left[\int_{t}^{T} \frac{1}{c(s)} g(r(x(s), c(s), s)) d s\right]
$$

where the term within the brackets is the total energy expenditure obtained as the integral of the power cost over time. The expectation above is taken over $\{c(s), s \in(t, T]\}$ and conditional on the starting state $x(t)=x, c(t)=c$. Define a minimum cost function, $J(x, c, t)$, as the infimum of $J_{r}(x, c, t)$ over the set of all admissible transmission policies.

$$
J(x, c, t)=\inf _{r(\cdot)} J_{r}(x, c, t), \quad r(x, c, t) \text { admissible }
$$

Thus, stated concisely, the optimization problem is to compute the minimum cost function $J(x, c, t)$ and obtain the optimal policy $r^{*}(x, c, t)$ that achieves this minimum cost.

\section{B. Optimality Conditions}

A standard approach towards studying continuous time problems is to investigate their behavior over a small time interval. In the context of the $B T$-problem, this methodology is summarized as follows. Suppose that the system is in state $(x, c, t)$. We first apply a transmission policy, $r(\cdot)$, in the small interval $[t, t+h]$ and thereafter, starting from the state $(x(t+h), c(t+h), t+h)$ we assume that the optimal policy is followed. By assumption, the energy cost is optimal over $[t+h, T]$, hence, investigating the system over $[t, t+h]$ would

\footnotetext{
${ }^{2}$ An additional technical requirement is that $r(x, c, t)$ be continuous and locally Lipschitz in $x$ (for $x>0$ ) which ensures that the ODE in (4) has a unique solution.
}

give conditions for the optimality of the chosen rate at time $t$. Since $t$ is arbitrary, we obtain formal conditions for an optimal policy.

Following the above approach, we now present the details of the analysis. Consider $t \in[0, T)$ and a small interval $[t, t+h]$, where $t+h<T$. Clearly, from Bellman's principle [23] the value function $J(x, c, t)$ satisfies,

$$
\begin{aligned}
J(x, c, t)= & \min _{r(\cdot)}\left\{E \int_{t}^{t+h} \frac{1}{c(s)} g(r(x(s), c(s), s)) d s\right. \\
& \left.+E J\left(x_{t+h}, c_{t+h}, t+h\right)\right\}
\end{aligned}
$$

where $x_{t+h}, c_{t+h}$ is a short-hand notation for $x(t+h)$ and $c(t+h)$ respectively. The expression within the curly brackets in (7) denotes the total cost with policy $r(\cdot)$ being followed over $[t, t+h]$ and the optimal policy thereafter. This cost must be clearly no less than the cost of applying the optimal policy directly from the starting state $(x, c, t)$. Thus for an admissible policy $r(\cdot)$ we obtain,

$$
\begin{aligned}
& J(x, c, t) \leq E \int_{t}^{t+h} \frac{1}{c(s)} g(r(x(s), c(s), s)) d s \\
&+E\left[J\left(x_{t+h}, c_{t+h}, t+h\right)\right] \\
& E\left[J\left(x_{t+h}, c_{t+h}, t+h\right)\right]-J(x, c, t) \\
&+E \int_{t}^{t+h} \frac{1}{c(s)} g(r(x(s), c(s), s)) d s \geq 0
\end{aligned}
$$

Dividing (9) by $h$ and taking the limit $h \downarrow 0$, we obtain,

$$
\mathcal{A}^{r} J(x, c, t)+\frac{1}{c} g(r) \geq 0
$$

since $\frac{1}{h} E \int_{t}^{t+h} \frac{g(r(x(s), c(s), s))}{c(s)} d s \rightarrow \frac{g(r)}{c} ; r$ is the transmission rate at time $t$, i.e. $r=r(x, c, t)$. The quantity $\mathcal{A}^{r} J(x, c, t)$ is defined as $\mathcal{A}^{r} J(x, c, t) \triangleq \lim _{h \downarrow 0} \frac{E J\left(x_{t+h}, c_{t+h}, t+h\right)-J(x, c, t)}{h}$.

The operator $\mathcal{A}^{r}$ is called the differential generator of the Markov process $(x(t), c(t))$ for the transmission policy $r(\cdot)$. Intuitively, it can be viewed as a natural generalization of the ordinary time derivative for a function that depends on a stochastic process. An elaborate discussion on this topic can be found in [23]-[25]. For the process $(x(t), c(t))$, using the time evolution in (4), the quantity $\mathcal{A}^{r} J(x, c, t)$ can be evaluated as [23],

$$
\begin{aligned}
\mathcal{A}^{r} J(x, c, t)= & \frac{\partial J(x, c, t)}{\partial t}-r(x, c, t) \frac{\partial J(x, c, t)}{\partial x} \\
& +\lambda\left(E_{z}[J(x, Z(c) c, t)]-J(x, c, t)\right)
\end{aligned}
$$

where $E_{z}$ is the expectation with respect to the $Z(c)$ variable as defined in (2).

Now, in the above steps from (8)-(10), if policy $r(\cdot)$ is replaced with the optimal policy $r^{*}(\cdot)$, equation (10) holds with equality, i.e.

$$
\mathcal{A}^{r^{*}} J(x, c, t)+\frac{1}{c} g\left(r^{*}\right)=0
$$

Thus, we see that for a given system state $(x, c, t)$, the optimal transmission rate $r^{*}$ is that value of $r$ that minimizes (10) and the minimum value of this expression equals zero. This gives,

$$
\min _{r \in[0, \infty)}\left[\frac{g(r)}{c}+\mathcal{A}^{r} J(x, c, t)\right]=0
$$


Substituting $\mathcal{A}^{r} J()$ from (11), we get a partial differential equation (PDE) in $J(x, c, t)$ which is also referred to as the Hamilton-Jacobi-Bellman (HJB) equation. This is the optimality equation for the $B T$-problem.

$$
\begin{aligned}
\min _{r \in[0, \infty)}\{ & \frac{g(r)}{c}+\frac{\partial J(x, c, t)}{\partial t}-r \frac{\partial J(x, c, t)}{\partial x} \\
& \left.+\lambda\left(E_{z}[J(x, Z(c) c, t)]-J(x, c, t)\right)\right\}=0
\end{aligned}
$$

The boundary conditions for the above PDE are, $J(0, c, t)=0$, and $J(x, c, T)=\infty$, if $x>0$. The last condition follows due to the deadline constraint of $T$ on the data.

While the above analysis gives the optimality equation, an important caveat is that it assumes $J(x, c, t)$ to be sufficiently smooth. Therefore, additionally, we also need converse arguments to verify that having a solution of (14) indeed gives the optimal solution. These technical details and the verification theorems are presented in Appendix A.

\section{Optimal Transmission Policy}

We have, so far, presented general results on the optimality condition for the $B T$-problem. We now give specific analytical results for the optimal policy and discuss some of the insights that can be drawn from it. However, before proceeding further a few additional notations regarding the channel process are required. Let there be total $m$ channel states in the Markov model and denote the various states $c \in \mathcal{C}$ as $c^{1}, c^{2}, \ldots, c^{m}$. Given a channel state $c^{i}$, the values taken by the random variable $Z\left(c^{i}\right)$ (defined in (2)) are denoted as $\left\{z_{i j}\right\}$, where $z_{i j}=c^{j} / c^{i}$. The probability that $Z\left(c^{i}\right)=z_{i j}$ is denoted as $p_{i j}$. Clearly, if there is no transition from state $c^{i}$ to $c^{j}, p_{i j}=0$. Also, without loss of generality we take the multiplicative constant $k=1$ in the function $g(r)=k r^{n}$ since any other value of $k$ simply scales the total cost in (5) but the optimal policy results remain the same.

Theorem I: Consider the BT-problem with $g(r)=r^{n}$, $n>1, n \in \mathbb{R}$ and a Markov channel model. The optimal policy, $r^{*}(x, c, t)$, and the minimum cost function, $J(x, c, t)$, are given by,

$$
\begin{aligned}
r^{*}\left(x, c^{i}, t\right) & =\frac{x}{f_{i}(T-t)}, i=1, \ldots, m \\
J\left(x, c^{i}, t\right) & =\frac{x^{n}}{c^{i}\left(f_{i}(T-t)\right)^{n-1}}, i=1, \ldots, m
\end{aligned}
$$

The functions $\left\{f_{i}(s)\right\}_{i=1}^{m}$ are the solution of the following ordinary differential equation $(O D E)$ system with the boundary conditions $f_{i}(0)=0, f_{i}^{\prime}(0)=1, \forall i^{3}$,

$$
\begin{aligned}
f_{1}^{\prime}(s) & =1+\frac{\lambda f_{1}(s)}{n-1}-\frac{\lambda}{n-1} \sum_{k=1}^{m} \frac{p_{1 k}}{z_{1 k}} \frac{\left(f_{1}(s)\right)^{n}}{\left(f_{k}(s)\right)^{n-1}} \\
& \vdots \\
f_{m}^{\prime}(s) & =1+\frac{\lambda f_{m}(s)}{n-1}-\frac{\lambda}{n-1} \sum_{k=1}^{m} \frac{p_{m k}}{z_{m k}} \frac{\left(f_{m}(s)\right)^{n}}{\left(f_{k}(s)\right)^{n-1}}
\end{aligned}
$$

\footnotetext{
${ }^{3}$ For numerical evaluation of the ODE solution, the two boundary conditions can be combined by taking a small $\epsilon>0$, letting $f_{i}(s)=s, s \in[0, \epsilon], \forall i$ and then using an initial-value ODE solver to obtain $\left\{f_{i}(s)\right\}, s \geq \epsilon$.
}

Proof: See Appendix B.

The results in the above theorem can be interpreted as follows. From (15), the optimal rate given $x$ amounts of data left, channel state $c^{i}$ and time $t$, is $\frac{x}{f_{i}(T-t)}$, where the function $f_{i}(s)$ is associated with the channel state $c^{i}$. The corresponding minimum expected cost starting from state $\left(x, c^{i}, t\right)$ is $\frac{x^{n}}{c^{i}\left(f_{i}(T-t)\right)^{n-1}}$. The boundary condition $f_{i}(0)=0$ is due to the deadline constraint, since at the deadline $(T-t)=0$ and we have $J\left(x, c^{i}, T\right)=\infty$, if $x \neq 0$. In full generality, the ODE system in (17)-(18) can be easily solved numerically using standard techniques (e.g. ODE solvers in MATLAB) and as shown in Appendix B, the system has a unique positive solution. Furthermore, this computation needs to be done only once before the system starts operating since the ODE system depends on the parameters of the channel model but not on the prevailing channel state. Thus, $\left\{f_{i}(s)\right\}$ can be pre-determined and stored in a table in the transmitter's memory. Once $\left\{f_{i}(s)\right\}$ are known, the closed form structure of the optimal policy in (15) warrants no further computation. At time $t$, the transmitter simply looks at the amount of data left in the queue, $x$, the channel state, $c^{i}$, and using the appropriate $f_{i}(\cdot)$ function it computes the transmission rate as $\frac{x}{f_{i}(T-t)}$.

The solution in (15) provides several interesting observations and insights as follows. At time $t$, the optimal rate depends on the channel state $c^{i}$ through the function $f_{i}(T-t)$ and this rate is linear in $x$ with slope $\frac{1}{f_{i}(T-t)}$. Thus, as intuitively expected, the rate is proportionately higher when there is more data left in the queue. Furthermore, we can view the quantity $\frac{1}{f_{i}(T-t)}$ as the "urgency" of transmission under the channel state $c^{i}$ and with $(T-t)$ time left until the deadline. This view gives a nice separation form for the optimal rate:

optimal rate $=$ amount of data left $*$ urgency of transmission

Due to the boundary condition, as $t$ approaches $T, f_{i}(T-t), \forall i$ goes to zero; thus, as expected, the urgency of transmission, $\frac{1}{f_{i}(T-t)}$, increases as $t$ approaches the deadline. Interestingly, if we set $\lambda=0$ (no channel variations) then, $f_{i}(T-t)=T-$ $t, \forall i$ and $r^{*}(x, c, t)=\frac{x}{T-t}$. Thus, with no channel variations the optimal policy is to transmit at a rate that just empties the queue by the deadline. This observation is consistent with the earlier results in the literature for non-fading/time-invariant channels [5], [7], [10]. We refer to this transmission scheme as the "Direct Drain" (DD) policy.

Simulation Example: Consider the two-state channel model with the states "bad" and "good" as described in Section II-B. Let $g(r)=r^{2}$ (i.e. $n=2$ ) and for simplicity take $\lambda_{b g}=\lambda_{g b}=$ $\lambda$. Denoting $\gamma=c_{b} / c_{g}$, we have, $Z\left(c_{g}\right)=\gamma$, w.p. 1 , and $Z\left(c_{b}\right)=1 / \gamma, w \cdot p$. 1. Denoting $f_{b}(s), f_{g}(s)$ as the respective functions in the bad and the good states, we have,

$$
\begin{aligned}
& f_{b}^{\prime}(s)=1+\lambda f_{b}(s)-\frac{\gamma \lambda\left(f_{b}(s)\right)^{2}}{f_{g}(s)} \\
& f_{g}^{\prime}(s)=1+\lambda f_{g}(s)-\frac{\lambda\left(f_{g}(s)\right)^{2}}{\gamma f_{b}(s)}
\end{aligned}
$$




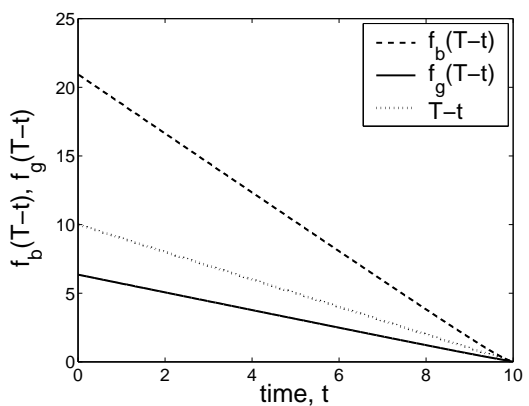

Fig. 5. $f_{b}(T-t)$ and $f_{g}(T-t)$ plot for the bad and the good channel respectively. The parameters are, $g(r)=r^{2}, T=10, \lambda=5, \gamma=0.3$.

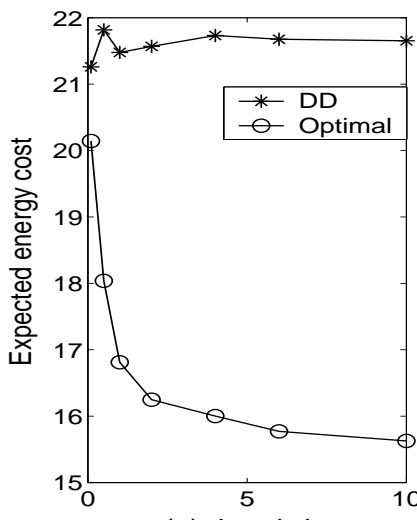

(a) Lambda

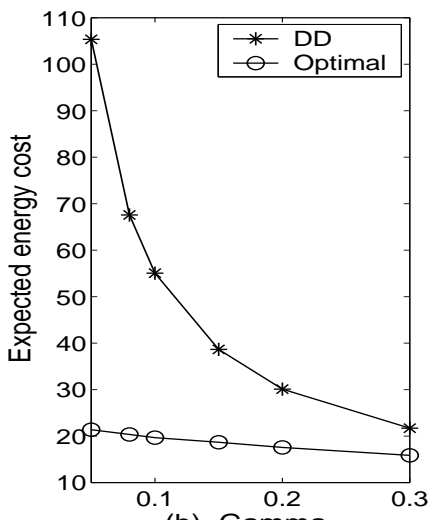

(b) Gamma

Fig. 6. Expected energy cost for the optimal and the direct drain (DD) policy.

Figure 5 plots these functions, evaluated using MATLAB, for $T=10, \lambda=5, \gamma=0.3$. First, as expected $f_{g}(T-t) \leq$ $f_{b}(T-t), \forall t$, which implies that given $x$ units of data in the buffer and time $t$, the rate $\frac{x}{f_{g}(T-t)}$ is higher under the good state than the bad state. Second, $f_{g}(T-t) \leq T-t \leq f_{b}(T-t)$, where the function, $T-t$, gives the rate, $\frac{x}{T-t}$, corresponding to the direct drain (DD) policy. Thus, the optimal policy both spreads the data over time and adapts the rate in response to the time-varying channel condition and this adaptation is governed by the respective functions $\left\{f_{i}(\cdot)\right\}$.

We now present illustrative simulation results to compare the performance of the optimal policy with the direct drain (DD) policy. As stated earlier, the DD policy transmits at a rate sufficient to just empty the buffer by the deadline without any rate adaptation to the channel state. For the simulations, we consider the two-state channel model with $c_{g}=1, c_{b}=\gamma$ and take $g(r)=r^{2}$. We let, $T=10$ and partition the interval $[0,10]$ into slots of length $d t=10^{-3}$, thus, having 10,000 time slots. The transmission rate chosen in each slot is obtained by evaluating the respective policies at the time corresponding to the start of that slot. A channel sample path is simulated using a Bernoulli process, where in a slot the channel transitions with probability $\lambda d t$ and with probability $1-\lambda d t$ there is no transition. At each transition, the new state is $\tilde{c}=Z(c) c$ which for the two-state model amounts to jumps between the two states. Expected energy cost is computed by taking an average over $10^{4}$ sample paths. Figure 6(a) plots the energy costs of the two policies as $\lambda$ is varied with $\gamma=0.3, B=10$.
When $\lambda$ is small the channel is essentially time-invariant over the deadline interval and the two policies are comparable. As $\lambda$ increases, the optimal cost substantially decreases due to the channel adaptation. In Figure 6(b), $\gamma$ is varied with $\lambda=5$, $B=10$. As $\gamma$ decreases the good and the bad channel quality differ significantly and the optimal rate adaptation leads to a substantially lower energy cost in terms of an order of magnitude difference as compared to the DD policy.

Constant Drift Channel: Theorem I gives the optimal policy for a general Markov channel model. By considering a special structure on the channel model which we refer to as the Constant Drift channel, two specialized results can be obtained. First, we obtain the $f(\cdot)$ function in closed form for the Monomial class $\left(g(r)=r^{n}\right)$, and second, we obtain the optimal policy for the Exponential class $\left(g(r)=\alpha^{r}-1\right)$.

In the constant drift channel model, we assume that the expected value of the random variable $1 / Z(c)$ is independent of the channel state, i.e. $E[1 / Z(c)]=\beta$, a constant. Thus, starting in state $c$, if $\tilde{c}$ denotes the next transition state we have $E\left[\frac{1}{\tilde{c}}\right]=E\left[\frac{1}{Z(c)}\right] \frac{1}{c}=\frac{\beta}{c}$. This means that if we look at the process $1 / c(t)$, the expected value of the next state is a constant multiple of the present state. We refer to $\beta$ as the "drift" parameter of the channel process. If $\beta>1$, the process $1 / c(t)$ has an upward drift; if $\beta=1$, there is no drift and if $\beta<1$, the drift is downwards. As a simple example of such a Markov model, suppose that the channel transitions at rate $\lambda>0$ and at every transition the state either improves by a factor $u>1$ with probability $p_{u}$, or worsens by a factor $1 / u$ with probability $p_{d}\left(=1-p_{u}\right)$. Thus, given some state $c>0$ the next channel state is either $u c$ or $c / u$, and, $E[1 / Z(c)]=$ $p_{u} / u+u p_{d}$. Here, the drift parameter $\beta=p_{u} / u+u p_{d}$.

The next theorem, Theorem II, gives the optimal policy result for the constant drift channel model and the monomial class of functions while Theorem III later gives the result for the exponential class.

Theorem II: Consider the BT-problem with $g(r)=r^{n}$, $n>1, n \in \mathbb{R}$ and a constant drift channel with drift $\beta$. The optimal policy, $r^{*}(x, c, t)$, and the function, $J(x, c, t)$, are,

$$
\begin{aligned}
r^{*}(x, c, t) & =\frac{x}{f(T-t)} \\
J(x, c, t) & =\frac{x^{n}}{c(f(T-t))^{n-1}}
\end{aligned}
$$

where $f(T-t)=\frac{(n-1)}{\lambda(\beta-1)}\left(1-\exp \left(-\frac{\lambda(\beta-1)}{n-1}(T-t)\right)\right)$.

Proof: See Appendix E.

The closed-form expression of $f(\cdot)$ above provides an interesting intuitive observation related to the parameter $\beta$. Suppose that the present channel state is $c$, then for a fixed rate $r$, the expected power cost in the next channel state is $E\left[\frac{g(r)}{Z(c) c}\right]=\frac{g(r)}{c} \beta$, which is $\beta$ times the present cost $\frac{g(r)}{c}$. This means that for higher values of parameter $\beta$, the channel on every transition drifts in an expected sense towards higher expected power cost or worsening conditions and vice-versa as $\beta$ decreases. Hence, as expected, the urgency of transmission $1 / f(t)$ is an increasing function with respect to $\beta$ since for 
large $\beta$ values it becomes more energy efficient to utilize the present channel conditions. Interestingly, when $\beta=1$, the expected future power cost does not change and in this case the optimal policy reduces to the direct drain (DD) policy, i.e. $r^{*}(x, c, t)=\frac{x}{T-t}$ (where we have used L'Hopital's rule to evaluate $f(\cdot)$ for $\beta=1$ ). Thus, we see that the direct drain policy is optimal both under no channel variations and under a constant drift channel model with $\beta=1$.

Theorem III: Consider the BT-problem with $g(r)=\alpha^{r}-$ $1, \alpha>1$ and a constant drift channel with drift $\beta$. The optimal policy, $r^{*}(x, c, t)$, is the following,

Case 1: $\beta \geq 1$,

$$
r^{*}(x, c, t)= \begin{cases}\sqrt{\frac{2 x \lambda(\beta-1)}{\ln \alpha}} & 0 \leq x<\frac{\lambda(\beta-1)(T-t)^{2}}{2 \ln \alpha}(23) \\ \frac{x}{T-t}+\frac{\lambda(\beta-1)(T-t)}{2 \ln \alpha} & x \geq \frac{\lambda(\beta-1)(T-t)^{2}}{2 \ln \alpha}\end{cases}
$$

Case 2: $0<\beta<1$,

$$
r^{*}(x, c, t)= \begin{cases}0 & 0 \leq x<\frac{\lambda(1-\beta)(T-t)^{2}}{2 \ln \alpha}(24) \\ \frac{x}{T-t}-\frac{\lambda(1-\beta)(T-t)}{2 \ln \alpha} & x \geq \frac{\lambda(1-\beta)(T-t)^{2}}{2 \ln \alpha}\end{cases}
$$

Proof: See Appendix F.

From above, we see that while the optimal rate function has a different functional form for the exponential case, some of the natural properties still hold true - it is monotonically increasing in $x$, increasing as $t$ approaches the deadline and also increasing in $\beta$.

\section{VARiable PaCKet Deadlines}

In the last section, we dealt with a specific case of the energy minimization problem involving $B$ bits of data and a single deadline $T$. We now extend the results to a more general setup where the data in the queue has variable deadlines. We adopt a cumulative curves methodology [3], [7], [22] and provide a natural decomposition of the problem in terms of multiple but dependent instances of the canonical BT-problem. Through this decomposition we obtain a transmission policy that is shown to be optimal for the constant drift channel model. As will be evident, the cumulative curves formulation provides a very appealing and simple visualization of the problem and the solution.

\section{A. Problem Setup}

Let us first define the following cumulative curves. Define the Arrival Curve, $A(t)$, as the total number of bits that have arrived to the queue in time $[0, t]$; the Departure Curve, $D(t)$, as the total number of bits that have departed (served) in time interval $[0, t]$ and the Minimum Departure Curve, $D_{\min }(t)$, as the minimum number of bits that must depart by time $t$ to satisfy the deadline constraints. For example, in the $B T$ problem case, we have $A(t)=B, t \in[0, T]$ since the queue has $B$ bits to begin with at time 0 and no more data is added. We have $D_{\min }(t)=0, t \in[0, T), D_{\min }(T)=B$ since until the deadline $t<T$ there is no minimum data transmission requirement while at $T$ the entire $B$ bits must have been transmitted. Finally, the curve $D(t)$ represents

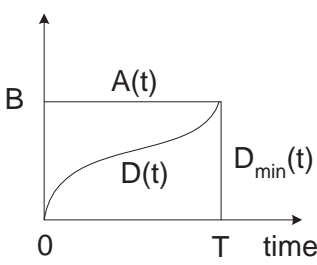

(a)

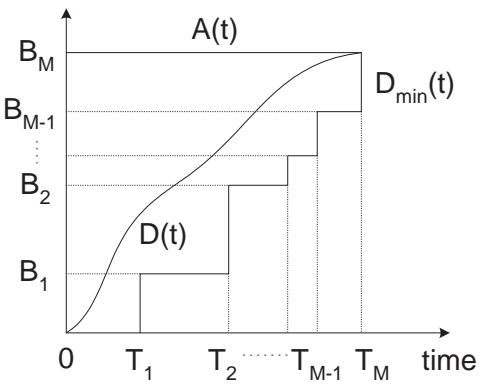

(b)
Fig. 7. Cumulative curves for (a) BT-problem, (b) Variable deadlines case.

the data departure over time which depends on the chosen transmission policy. A schematic diagram of this is given in Figure 7(a).

Consider now the variable deadlines problem. Here, the queue has $M$ packets that are arranged and served in the earliest-deadline-first order. Let $b_{j}$ be the number of bits in the $j^{\text {th }}$ packet and $T_{j}$ be the deadline for this packet; assume $0<T_{1}<T_{2}<\ldots<T_{M}$. There are no new arrivals and the objective is to obtain a transmission policy that serves this data over the time-varying channel with minimum expected energy cost while meeting the deadline constraints. In terms of the cumulative curves, the setup can be visualized as depicted in Figure 7(b). Let $B_{j}=\sum_{l=1}^{j} b_{l}$; where $B_{j}$ is the cumulative amount of data of the first $j$ packets. Then, $A(t)=B_{M}, \forall t$, since a total $B_{M}$ bits are in the queue at time 0 and no more data is added, and, $D_{\min }(t)$ is a piecewise-constant curve with jumps at times $T_{j}$, i.e. at time $T_{j}, D_{\min }\left(T_{j}\right)=B_{j}$ since the first $B_{j}$ bits must be transmitted by $T_{j}$. Finally, we require that for admissibility a transmission policy must be such that the departure curve, $D(t)$, satisfy $D_{\min }(t) \leq D(t) \leq A(t)$; in other words, data must be served such that the cumulative amount lies above the minimum departure curve (deadline constraints) and below the arrival curve (causality constraints). Note that if a transmission policy adapts the rate with the channel variations, the actual departure curve followed would depend on the underlying channel sample path; however, for all the sample paths (almost surely) the above admissibility criterion must be satisfied.

\section{B. Optimal Control Formulation}

Let the system state be denoted as $(D, c, t)$, where the notation means that at the present time $t$, the cumulative amount of data that has been transmitted is $D(t)=D$, and the channel state is $c(t)=c$. Let $r(D, c, t)$ denote a transmission policy and since the underlying channel process is Markov, it is again sufficient to restrict attention to policies that depend only on the present system state [25]. Furthermore, we will assume that the function $g(r)$ belongs to the Monomial class, i.e. $g(r)=k r^{n}, k>0, n>1$.

As before, given a policy $r(D, c, t)$ the system evolves in time as a Piecewise-Deterministic-Process starting in the initial state $D(0)=0$ and $c(0)=c_{0}$. Until $\tau_{1}$, where $\tau_{1}$ is the first time instant after $t=0$ at which the channel changes, data is 
transmitted at the rate $r\left(D(t), c_{0}, t\right)$. Hence, over the interval $\left[0, \tau_{1}\right), D(t)$ satisfies the differential equation,

$$
\frac{d D(t)}{d t}=r\left(D(t), c_{0}, t\right)
$$

Equivalently, in integral form $D(t)=D(0)+$ $\int_{0}^{t} r\left(D(s), c_{0}, s\right) d s, t \in\left[0, \tau_{1}\right]$. Then, starting from the new state $\left(D\left(\tau_{1}\right), c\left(\tau_{1}\right), \tau_{1}\right)$ until the next channel transition we have, $\frac{d D(t)}{d t}=r\left(D(t), c\left(\tau_{1}\right), t\right), t \in\left[\tau_{1}, \tau_{2}\right)$; and this procedure repeats until $t=T_{M}$ is reached.

A transmission policy, $r(D, c, t)$, is admissible, if it satisfies the following: (a) $0 \leq r(D, c, t)<\infty$, (non-negativity), and, (b) $D_{\min }(t) \leq D(t) \leq A(t), t \in\left[0, T_{M}\right]$, a.s. (deadline and causality constraints).

For an admissible policy $r(\cdot)$, let $J_{r}(D, c, t)$ denote the cost-to-go function as the expected energy expenditure starting from an admissible state $(D, c, t)$, (i.e. $D_{\min }(t) \leq D \leq$ $\left.A(t), t<T_{M}\right)$. Then,

$$
J_{r}(D, c, t)=E\left[\int_{t}^{T_{M}} \frac{1}{c(s)} g(r(D(s), c(s), s)) d s\right]
$$

where the above expectation is taken over $\left\{c(s), s \in\left(t, T_{M}\right]\right\}$ and conditional on the starting state $D(t)=D, c(t)=c$. Define a minimum cost function, $J(D, c, t)$, as the infimum of $J_{r}(D, c, t)$ over the set of all admissible transmission policies.

$$
J(D, c, t)=\inf _{r(\cdot)} J_{r}(D, c, t), \quad r(D, c, t) \text { admissible }
$$

As before, the optimization problem is to compute the optimal policy $r^{*}(D, c, t)$ that achieves the minimum cost $J(D, c, t)$.

Following Section III-B, the optimality HJB equation can be obtained directly by noting that the process evolution as discussed above remains the same as the $B T$-problem, except that, for convenience we have used the cumulative data transmitted $D(t)$ as the state variable. Thus, using the results of Section III-B the HJB equation is given as,

$$
\begin{aligned}
\min _{r \in[0, \infty)}\left\{\frac{g(r)}{c}+\frac{\partial J(D, c, t)}{\partial t}+r \frac{\partial J(D, c, t)}{\partial D}\right. \\
\left.+\lambda\left(E_{z}[J(D, Z(c) c, t)]-J(D, c, t)\right)\right\}=0
\end{aligned}
$$

However, the boundary conditions for the above general formulation are more complex than the $B T$-problem and depend on the underlying $D_{\min }(t)$ curve. For the variable deadlines setup, these are given as, $J\left(B_{M}, c, t\right)=0$ and $J\left(D, c, T_{j}\right)=\infty$, if $D<B_{j}, j=1, \ldots, m$. The second condition follows from the deadline constraints since as $t$ approaches $T_{j}$ the cost function must be unbounded if the required cumulative amount $B_{j}$ has not been transmitted.

\section{Transmission Policy}

A direct solution of the optimization problem stated in the previous section is fairly difficult due to the complexity of solving the differential equation with multiple boundary conditions involved. Interestingly, however, the simple graphical visualization of cumulative curves provides an intuitive and natural decomposition of the variable deadlines problem in terms of multiple inter-related $B T$-problems. A visual comparison of the two diagrams in Figure 7 suggests the following approach. First, instead of looking at individual packets in the queue, we can visualize the deadline constraints in terms of cumulative amounts as $\left\{B_{j} T_{j}\right\}_{j=1}^{M}$ constraints, that is, a total of $B_{j}$ bits must be transmitted by deadline $T_{j}(j=1, \ldots, M)$. Clearly, each individual $B_{j} T_{j}$ constraint is like a $B T$-problem except that now there are multiple such constraints that all need to be satisfied. For every time $t$ and channel state $c$, we know the optimal transmission rate to satisfy each of the $B_{j} T_{j}$ constraints individually (assuming only this constraint existed), thus, to meet all the constraints a natural solution is to simply choose the maximum rate; i.e. procedurally, for a given system state list the remaining $B_{j} T_{j}$ constraints, obtain the transmission rate individually for each one of them using the rate function obtained previously for the $B T$-problem, and then choose the maximum value.

More precisely, this transmission policy is described as follows. Let the system be in state $(D, c, t)$ and consider a particular $B_{j} T_{j}$ constraint. Using (15), the optimal rate for an individual $B_{j} T_{j}$ constraint for channel state $c^{i}$ is given as $\frac{B_{j}-D}{f_{i}\left(T_{j}-t\right)}$, since $\left(B_{j}-D\right)$ is the amount of data left and $\left(T_{j}-t\right)$ is the time left until the deadline $T_{j}$. Let $\tilde{r}(D, c, t)$ denote the transmission rate for our proposed policy, then $\tilde{r}(\cdot)$ is the maximum value among the rates for all $B_{j} T_{j}$ constraints for which $\left(B_{j} \geq D, T_{j} \geq t\right)$; i.e.

$$
\tilde{r}\left(D, c^{i}, t\right)=\max _{j:\left(B_{j} \geq D, T_{j} \geq t\right)} \frac{B_{j}-D}{f_{i}\left(T_{j}-t\right)}
$$

where, as before, the functions $\left\{f_{i}(s)\right\}_{i=1}^{m}$ are the solution of the following ODE system with the boundary conditions $f_{i}(0)=0, f_{i}^{\prime}(0)=1, \forall i$,

$f_{i}^{\prime}(s)=1+\frac{\lambda f_{i}(s)}{n-1}-\frac{\lambda}{n-1} \sum_{k=1}^{m} \frac{p_{i k}}{z_{i k}} \frac{\left(f_{i}(s)\right)^{n}}{\left(f_{k}(s)\right)^{n-1}}, i=1, \ldots, m$

By construction all the $B_{j} T_{j}$ constraints are satisfied since at all times we choose the maximum rate among those needed to meet each of the remaining constraints. Hence, the policy in (29) is admissible. Furthermore, since the policy in (29) is based on the $B T$-solution, it inherits all the properties of that solution. The ODE system in (29) is identical to the $B T$ case, hence, as before the functions $\left\{f_{i}(s)\right\}_{i=1}^{m}$ can be obtained numerically using a standard ODE solver. This computation needs to be done only once before the system starts operating and the functions $\left\{f_{i}(s)\right\}$ can be pre-determined and stored in a table in the transmitter's memory. Once the $\left\{f_{i}(s)\right\}$ are known, the online computation is minimal. At time $t$, the transmitter looks at the cumulative amount of data transmitted, $D$, the channel state, $c^{i}$, and then using the corresponding $f_{i}(\cdot)$ function it simply computes the maximum among a set of values as given in (29).

While the transmission policy in (29) applies for a general Markov channel model, under the specialization to a constant drift channel it is in fact the optimal policy as shown in the following theorem. Note that for the constant drift channel model, the function $f_{i}(s)=f(s), \forall i$ where $f(\cdot)$ is given in Theorem II. 
Theorem IV: (Variable Deadlines Case) Consider the variable deadlines problem with $g(r)=r^{n}, n>1, n \in \mathbb{R}$ and the constant drift channel model with parameter $\beta$. The optimal rate, $r^{*}(D, c, t)$ for $D_{\min }(t) \leq D \leq A(t), t \in\left[0, T_{M}\right)$ is given as,

$$
r^{*}(D, c, t)=\max _{j:\left(B_{j} \geq D, T_{j} \geq t\right)} \frac{B_{j}-D}{f\left(T_{j}-t\right)}
$$

where, $f(s)=\frac{(n-1)}{\lambda(\beta-1)}\left(1-\exp \left(-\frac{\lambda(\beta-1)}{n-1} s\right)\right)$.

Proof: See Appendix G.

\section{Packet Arrivals with Deadlines}

Consider an arbitrary stream of packet arrivals to the queue with each packet having a deadline by which it must depart. Regardless of the underlying stochastic process generating the packets, we next present a heuristic energy-efficient transmission policy based on the variable deadlines solution. We call it the "BT-Adaptive" (BTA) policy. Since this policy is not based on a specific arrival process, it is robust to changes in the arrival statistics and can accommodate multiple deadline classes of packet arrivals to the queue. Finally, to evaluate the performance of the BTA policy, we also present illustrative simulation results comparing it with a non-adaptive scheme.

\section{A. BT-Adaptive (BTA) Policy}

Consider packet arrivals to the queue and assume that the arrivals occur at discrete times with each packet having a deadline associated with it. Clearly, at the instant immediately following a packet arrival the transmitter queue consists of, (a) earlier remaining packets with their deadlines and (b) the new packet with its own deadline. Re-arranging the data in the queue in the earliest-deadline-first order we can view the queue as consisting of some total amount $B_{M}$ of data with variable deadlines, identical to the case considered in the last section. Not assuming any knowledge of the future arrivals and using (29) we have an energy efficient policy to empty the transmitter buffer. As this policy is followed, at the next packet arrival instance the above procedure is simply repeated by updating the data amount taking into account the new packet.

Summarizing, the BTA policy is as follows: Transmit the data in the queue with the rate as given in (29); at every packet arrival instant re-arrange the data in the earliest-deadline-first order to obtain a new set of $B_{j} T_{j}$ values including the new packet and its deadline; re-initialize D to zero and follow (29) thereafter.

\section{B. Simulation Results}

In this section, we present simulation results to evaluate the performance of the BT-Adaptive policy. For comparison purposes we consider the "Head-of-Line Drain" (HLD) policy which can be easily implemented in practice. In HLD policy, the data in the queue is arranged in the earliest-deadline-first order and the packets are served in that order. At time $t$, let $H_{t}$ be the amount of data left in the head-of-the-line packet and $T_{H}$ be the amount of time until its deadline, then the rate chosen is $r_{t}=\frac{H_{t}}{T_{H}}$. Thus, the transmitter serves the first packet

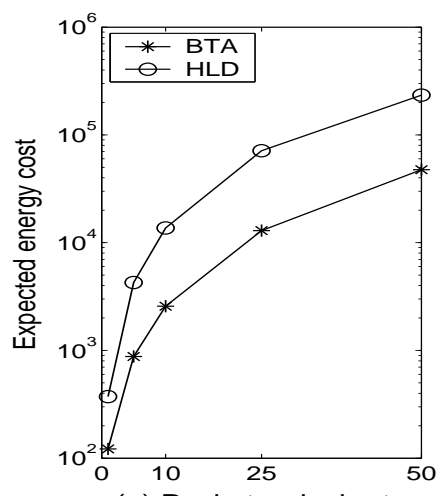

(a) Packet arrival rate

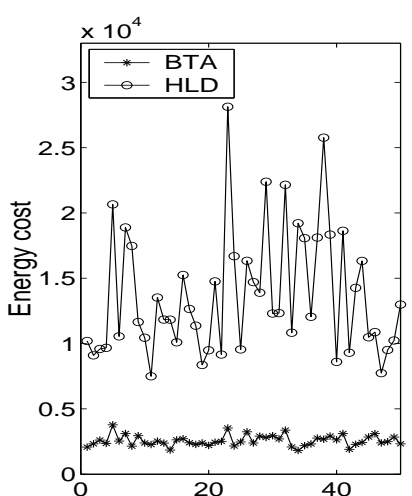

(b) Sample path index
Fig. 8. Energy cost comparison for Poisson arrival process for (a) different arrival rate, (b) different sample paths.

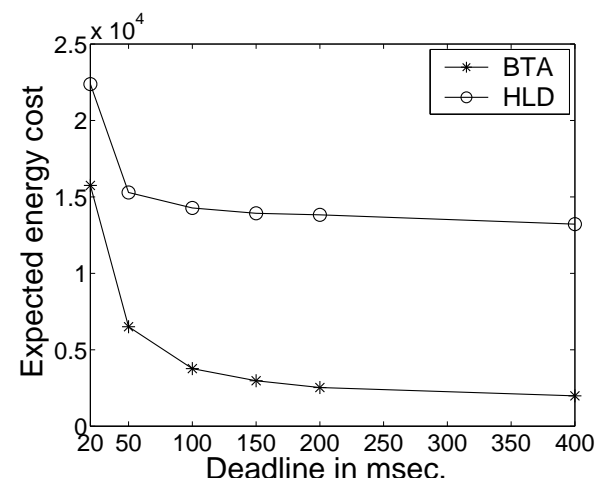

Fig. 9. Energy cost versus packet deadline for Poisson arrival process.

in queue at a rate to transmit it out by its deadline, then moves to the next packet in line and so on. At every packet arrival instant, the data in the queue is re-arranged in the earliestdeadline-first order and the above policy is repeated with the new packet taken into account.

The simulation setup is as follows. The queue has packet arrivals and each packet has a deadline associated with it. On each simulation run, the total time over which the packets arrive and the system is operated is taken as $L=10$ seconds. This interval $[0,10]$ is partitioned into 10,000 slots, thus each slot is of duration $d t=1 \mathrm{msec}$. The channel model is the two state model, described in Section II-B with the parameters, $c_{g}=1, c_{b}=0.2, \lambda_{b g}=\lambda_{g b}=\lambda=50$. Thus, the average time spent in a state before the channel transitions is $1 / 50$ seconds, or $20 \mathrm{msec}$. A channel sample path is simulated using a Bernoulli process where in a slot the channel transitions with probability $\lambda d t$; otherwise there is no transition. For simplicity, the packet arrival and the channel state transitions occur only at the slot boundaries. For both the BTA and the HLD policies, the rate chosen in a slot is obtained by evaluating the respective policies at the time corresponding to the start of that slot. We take $g(r)=r^{2}$; energy cost per slot is therefore $\frac{r^{2} d t}{c}(r$ is the chosen rate in the slot) and the total expected energy cost is taken as an average of the total cost over multiple sample runs.

We first consider a Poisson packet arrival process with each packet having 1 unit of data and a deadline of $200 \mathrm{msec}$. 


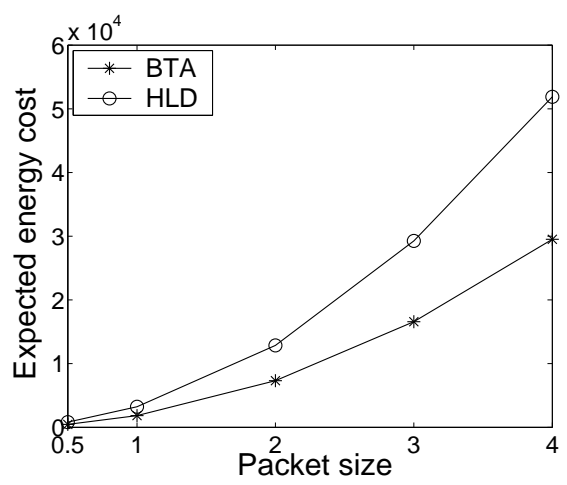

Fig. 10. Energy cost versus packet size for Uniform arrival process.

Figure 8(a) is a plot of the expected energy cost, plotted on a $\log$ scale, versus the packet arrival rate. Note that a packet arrival rate of 10 implies that the average inter-arrival time of a packet is $1 / 10 \mathrm{sec}$. or $100 \mathrm{msec}$. As is evident from the plot, the BTA policy has a much lower energy cost compared to the HLD policy and as the arrival rate increases the two costs are roughly an order of magnitude apart. This can be intuitively explained as follows. When the arrival rate is low, most of the time the queue has at most a single packet. Hence, both policies choose a rate based on the head-of-line packet with the BTA policy also adapting the rate with the channel state. As the arrival rate increases and due to the bursty nature of the Poisson process, the queue tends to have more packets. The BTA policy then adapts based on the channel and the deadlines of all the packets in the queue, whereas, the HLD policy chooses a rate based solely on the head-of-line packet. The energy efficiency of the BTA policy is not just in an average sense but even on individual sample paths. This is shown in Figure 8(b) for 50 sample paths for arrival rate of 10 packets/second.

In Figure 9, the packet arrival process is Poisson with rate 10 but now the packet deadline is varied. Clearly, as seen in the figure, the energy cost decreases as the packet deadline increases since lower transmission rates are required to meet the deadlines. Also, as the deadline increases the energy cost difference between the BTA and the HLD policy increases. This is because with a larger delay constraint there is more room for the adaptive techniques employed in the BTA policy to have a greater effect.

In Figure 10, we consider a Uniform packet arrival process where now the inter-arrival time between packets is uniformly distributed between 50 and $150 \mathrm{msec}$. The deadline for each packet is taken as $200 \mathrm{msec}$ while the packet size is varied. First, as expected the energy cost for both the policies increases with the packet size and second, the BTA policy has a much less energy cost compared with HLD policy even when the arrival process is less bursty as compared to the Poisson process.

\section{CONCLUSION}

We considered transmission of delay-constrained data over time-varying channels with the objective of minimizing the total transmission energy expenditure. We adopted a novel approach based on continuous-time formulation and stochastic control theory to address an otherwise difficult set of problems. We first considered the problem of transmitting $B$ units of data by deadline $T$ and obtained the optimal rate adaptation policy. Various properties of the optimal rate function are deduced and it is also shown to have an intuitive separation form. Using a cumulative curves methodology and a decomposition approach, we then obtained an energy-efficient rate control policy when the data in the queue has variable deadline constraints; this policy is shown to be optimal under specific scenarios. Finally, based on the intuition developed in the above, we devised a heuristic policy for arbitrary packet arrival process and compared its performance through simulations. We believe that the framework of this paper holds promise for various extensions addressing QoS-constrained data transmission in wireless systems. Some of the natural extensions include a network model with multiple transmitter-receiver pairs and multi-hop transmissions with end-to-end delay constraints.

\section{ACKNOWLEDGEMENTS}

The authors would like to acknowledge John Wyatt for helpful technical discussions on the work.

\section{REFERENCES}

[1] A. Jalali, R. Padovani and R. Pankaj, "Data throughput of CDMA-HDR a high efficiency high data rate personal communication wireless system", IEEE Vehicular Technology Conf., vol. 3, 2000.

[2] L. Tsaur and D. Lee, "Closed-loop architecture and protocols for rapid dynamic spreading gain adaptation in CDMA networks",INFOCOM, 2004

[3] Murtaza Zafer, "Dynamic Rate-Control and Scheduling Algorithms for Quality-of-Service in Wireless Networks", PhD Thesis, Massachusetts Institute of Technology (MIT), Sept. 2007.

[4] R. Berry and R. Gallager, "Communication over fading channels with delay constraints", IEEE Tran. on Information Theory, May 2002.

[5] E. Uysal-Biyikoglu and A. El Gamal, "On Adaptive transmission for energy-efficiency in wireless data networks," in IEEE Trans. Information Theory, December 2004.

[6] A. Fu, E. Modiano and J. Tsitsiklis, "Optimal energy allocation for delay constrained data transmission over a time-varying channel", IEEE INFOCOM 2003, vol. 2, pp 1095 - 1105, April 2003.

[7] M. Zafer and E. Modiano, "A Calculus approach to minimum energy transmission policies with quality of service guarantees", IEEE INFOCOM 2005, March 2005.

[8] M. Zafer and E. Modiano, "Continuous-time optimal rate control for delay constrained data transmission", 43rd Allerton conf. on Communication, Control and Computing, Monticello, Sept. 2005.

[9] M. Zafer and E. Modiano, "Minimum energy transmission over a wireless fading channel with packet deadlines", IEEE CDC, New Orleans, Dec. 2007.

[10] M. Khojastepour and A. Sabharwal, "Delay-constrained scheduling: power efficiency, filter design and bounds", IEEE INFOCOM, March 2004

[11] P. Nuggehalli, V. Srinivasan and R. Rao, "Delay constrained energy efficient transmission strategies for wireless devices", IEEE INFOCOM 2002, vol. 3 , pp. 1765 - 1772,June 2002.

[12] M. Neely, E. Modiano and C. Rohrs, "Dynamic power allocation and routing for time varying wireless networks," IEEE INFOCOM, 2003.

[13] A. Goldsmith, "The capacity of downlink fading channels with variable rate and power", IEEE Tran. Vehicular Technology, vol. 45, Oct. 1997.

[14] L. Tassiulas and A. Ephremides, "Stability properties of constrained queueing systems and scheduling policies for maximum throughput in multihop radio networks", IEEE Tran. on Automatic Control, Dec. 1992.

[15] X. Liu, E. Chong and N. Shroff, "A framework for opportunistic scheduling in wireless networks " Computer Networks, vol. 41, 2003.

[16] B. Collins and R. Cruz, "Transmission policies for time varying channels with average delay constraints", Proceedings, 1999 Allerton conf. on Communication, Control and Computing, Monticello, IL, 1999. 
[17] B. Ata, "Dynamic power control in a wireless static channel subject to a quality of service constraint", Operations Research 53 (2005), No 5, 842-851.

[18] F. Babich and G. Lombardi, "A Markov model for the mobile propagation channel", IEEE Tran. on Vehicular Technology, vol. 49, no. 1, pp. 63-73, Jan. 2000.

[19] Q. Zhang and S. Kassam, "Finite-State Markov Model for Rayleigh Fading Channels", IEEE Tran. on Communications, vol. 47, no. 11, Nov. 1999.

[20] E. N. Gilbert, "Capacity of burst-noise channel”, Bell Syst. Tech. J.,vol. 39, no. 9, pp. 1253-1265, Sept. 1960.

[21] A. Goldsmith and P. Varaiya, "Capacity, mutual Information and coding for finite state markov channels", IEEE Trans. Information Theory, 1996.

[22] J. Le Boudec, P. Thiran, Network calculus, Springer-Verlag, 2001.

[23] W. Fleming and H. Soner, Controlled Markov Processes and Viscosity Solutions, Springer-Verlag, 1993.

[24] M. Davis, Markov Models and Optimization, Chapman and Hall, 1993

[25] B. Oksendal, Stochastic Differential Equations, Springer, $5^{\text {th }}$ edn., 2000.

[26] J. Cronin, Differential Equations: Introduction and Qualitative Theory, New York: Marcel Dekker, Inc., 1980.

[27] W. Rudin, Principles of Mathematical Analysis, McGraw-Hill, $3^{\text {rd }}$ edition, Singapore, 1976.

\section{APPENDIX A}

\section{VERIFICATION THEOREM FOR THE $B T$-PROBLEM}

In Section III-B, we obtained heuristically the optimality equation as given by (14). To present a rigorous argument we need to verify that a solution of (14), i.e. functional forms $J(x, c, t)$ and $r^{*}(x, c, t)$ that satisfy (14), indeed give the optimal solution for the $B T$-problem. However, the standard verification theorems in [23] that provide conditions to check for the optimality of the solution to the HJB equation do not directly apply for the $B T$-problem. This is because the nonstandard boundary condition $x(T)=0$ leads to a singularity in $J(x, c, t)$ at $t=T$ (since, $J(x, c, t) \stackrel{(t \rightarrow T)}{\longrightarrow} \infty$, if $x>0$ ). To overcome this technical difficulty and obtain a verification theorem for the $B T$-problem, we consider a particular relaxation and take appropriate limits as discussed next.

Consider the following modification to the problem. Instead of emptying the buffer by time $T$, extend the deadline to $T+\tau_{k}$ for some $\tau_{k}>0$. In the interval $\left[T, T+\tau_{k}\right]$ the channel does not change and whatever data, $x(T)$, left at time $T$ is transmitted out at the constant rate $x(T) / \tau_{k}$. Thus, now the system runs over time $[0, T]$ and the data left at $T$ has a terminal energy cost of emptying it in the next $\tau_{k}$ interval. This terminal cost is given as,

$$
h_{k}(x(T), c(T))=\frac{\tau_{k} g\left(\frac{x(T)}{\tau_{k}}\right)}{c(T)}
$$

We now consider a sequence $\left\{\tau_{k}\right\}_{1}^{\infty}$ such that $\tau_{k} \downarrow 0$. This gives a sequence of modified problems which we denote as $\left\{\mathcal{P}_{k}\right\}$ while the corresponding minimum-cost functions are denoted as $\left\{J^{k}(x, c, t)\right\}$.

Note that the relaxation does not change the system dynamics over time but only affects the terminal cost applied to the leftover data at time $T$. In the $B T$-problem, we had an infinite cost on any data left at $T$ but now each problem $\left\{\mathcal{P}_{k}\right\}$ has a smooth function $h_{k}(x(T), c(T))$ associated with it. Clearly, then, the optimality equation for each $\mathcal{P}_{k}$ is the same as (14) except that the boundary conditions for the PDE now become $J^{k}(0, c, t)=0$ and $J^{k}(x, c, T)=h_{k}(x, c)$. The admissibility of a policy for problem $\mathcal{P}_{k}$ includes the constraints required for the $B T$-problem with the exception of $x(T)=0$ which is no longer a necessary requirement. Furthermore, from the increasing and convexity properties of $g(r)$, it is easy to see that for a fixed $(x, c), h_{k}(x, c)=0$, if $x=0, \forall k$ and $h_{k}(x, c) \stackrel{(k \rightarrow \infty)}{\longrightarrow} \infty$, if $x>0$. Thus, as we look at the modified problems $\mathcal{P}_{k}$ with large values of $k$ (smaller values of $\tau_{k}$ ), there is an increasingly higher penalty cost applied to the data left at time $T$. And as $k \rightarrow \infty$, this penalty cost goes to infinity; thus, in the limit we have a situation equivalent to the $B T$-problem. The rest of the proof delves into the technical details involved in taking the limits. Specifically, to obtain the verification theorem for the $B T$-problem, we show that having obtained the optimal cost function for the modified problem $\mathcal{P}_{k}$ and then taking the limit $k \rightarrow \infty$ gives the optimal solution for the $B T$-problem.

We will use the notation $\tilde{\Gamma}$ to denote the set of all admissible policies for problem $\mathcal{P}_{k}$ (note that for all $\mathcal{P}_{k}$, the set $\tilde{\Gamma}$ is the same since the problems only differ in the terminal cost function $\left.h_{k}(\cdot, \cdot)\right)$. The cost-to-go function for a policy $r(\cdot)$ for problem $\mathcal{P}_{k}$ will be denoted as $J_{r}^{k}(x, c, t)$; i.e. $J_{r}^{k}(x, c, t)=E\left[\int_{t}^{T} \frac{g(r(x(s), c(s), s))}{c(s)} d s+h_{k}(x(T), c(T))\right]$. We start with Lemma 1 which gives the verification result for problem $\mathcal{P}_{k}$. It states that a solution of the PDE equation (14) satisfying the relevant boundary conditions indeed gives the minimum cost function and that the transmission policy obtained from the minimizing $r$ in (14) is the optimal policy.

Lemma 1: (Verification Result for $\left.\mathcal{P}_{k}\right)$ Let $J^{k}(x, c, t)$ defined on $[0, B] \times \mathcal{C} \times[0, T]$, solve the equation in (14) with the boundary conditions $J^{k}(0, c, t)=0, \forall c \in \mathcal{C}, t \in[0, T)$ and $J^{k}(x, c, T)=h_{k}(x, c)$. Then,

1) $J^{k}(x, c, t) \leq J_{r}^{k}(x, c, t), \quad \forall r(\cdot) \in \tilde{\Gamma}$

2) Let $r_{k}^{*}(x, c, t)$ be an admissible policy for $\mathcal{P}_{k}$ such that $r_{k}^{*}$ is the minimizing value of $r$ in (14) for $J^{k}(x, c, t)$, then, $r_{k}^{*}(x, c, t)$ is an optimal policy, $J^{k}(x, c, t)$ is the minimum cost function and,

$$
\begin{gathered}
J^{k}(x, c, t)=E\left[\int_{t}^{T} \frac{g\left(r_{k}^{*}(x(s), c(s), s)\right)}{c(s)} d s\right. \\
\left.+h_{k}(x(T), c(T))\right]
\end{gathered}
$$

Proof: See [23], Chap III, Theorem 8.1.

Now, define the function $J(x, c, t) \triangleq \lim _{k \rightarrow \infty} J^{k}(x, c, t)$. The next theorem shows that this limit exists and if it satisfies (14), it is the optimal solution for the $B T$-problem. We will use the notation $\Gamma$ in the theorem to denote the set of all admissible policies for the $B T$-problem.

Theorem V: (Verification for the BT-problem) Consider $(x, c, t) \in[0, B] \times \mathcal{C} \times[0, T)$ and define $J(x, c, t) \triangleq \lim _{k \rightarrow \infty} J^{k}(x, c, t)$. Let $J(x, c, t)$ satisfy the $H J B$ equation in (14) and $r^{*}(x, c, t)$ be an admissible policy for the BT-problem such that $r^{*}$ is the minimizing value of $r$ in (14) for $J(x, c, t)$. Then,

1) $J(x, c, t) \leq J_{r}(x, c, t), \quad \forall r(\cdot) \in \Gamma$

2) $r^{*}(x, c, t)$ is the optimal policy, $J(x, c, t)$ is the minimum 
cost function and,

$$
J(x, c, t)=E\left[\int_{t}^{T} \frac{1}{c(s)} g\left(r^{*}(x(s), c(s), s)\right) d s\right]
$$

Proof: We divide the proof into various steps each giving arguments for the various claims in the theorem statement.

Step 1: The limit, $J(x, c, t)=\lim _{k \rightarrow \infty} J^{k}(x, c, t)$ exists and is finite

Consider the relaxed problem $\mathcal{P}_{k}$ and the corresponding minimum cost function $J^{k}(x, c, t)$. We now make two claims, first that $J^{k}(x, c, t)$ is non-decreasing in $k$ for each $(x, c, t)$ and second that $J^{k}(x, c, t)$ is bounded for all $k$. These two claims are proved as follows. First, note that the sequence $\tau_{k}$ is decreasing and hence $h_{k}(x, c)$ is monotonically point-wise increasing in $x$ with increasing $k$. Fix an admissible policy $r(\cdot) \in \tilde{\Gamma}$, then for every channel sample path the total energy cost is higher as $k$ increases because the terminal cost is higher. Hence, for all $r(\cdot)$ the expected energy cost increases with $k$; taking the infimum over $r(\cdot)$ proves the first claim. To prove the second claim consider a simple policy, $\pi(\cdot)$, that starting with $x$ units of data at time $t$, empties this data at a constant rate by time $\tilde{t}$, where $t<\tilde{t}<T$; i.e. $r=x /(\tilde{t}-t)$ is fixed. For such a policy,

$$
\begin{aligned}
J_{\pi}(x, c, t) & =E\left[\int_{t}^{\tilde{t}} \frac{g(x /(\tilde{t}-t))}{c(s)} d s\right] \\
& =g\left(\frac{x}{\tilde{t}-t}\right) E\left[\int_{t}^{\tilde{t}} \frac{1}{c(s)} d s\right] \\
& \leq g\left(\frac{x}{\tilde{t}-t}\right) \sum_{j=0}^{\infty}\left(\frac{(\tilde{t}-t)}{c\left(z_{l}\right)^{j}}\right) \frac{(\lambda(\tilde{t}-t))^{j} e^{-\lambda(\tilde{t}-t)}}{j !} \\
& =\frac{(\tilde{t}-t)}{c} g\left(\frac{x}{\tilde{t}-t}\right) e^{\frac{\lambda(\tilde{t}-t)}{z_{l}}} e^{-\lambda(\tilde{t}-t)}<\infty
\end{aligned}
$$

The inequality above follows by first conditioning that the channel makes $j$ transitions over $[t, \tilde{t}]$, taking $c(s)=\left(z_{l}\right)^{j} c$, where $\left(z_{l}\right)^{j} c$ is the worst possible channel quality starting with state $c$ and making $j$ transitions, and finally taking expectation with respect to $j$ (number of transitions, $j$, is Poisson distributed with rate $\lambda(\tilde{t}-t)$ and $z_{l}>0$ is the least value that any $Z(c)$ can take). Since, $J^{k}(x, c, t) \leq J_{\pi}(x, c, t), \forall k$, the bounded-ness claim follows. Combining the above two claims (non-decreasing and bounded), we see that the point-wise limit $J(x, c, t)=\lim _{k \rightarrow \infty} J^{k}(x, c, t)$ exists.

Step 2: Result 1 stated in the theorem, i.e. $J(x, c, t) \leq$ $J_{r}(x, c, t), \forall B T$-admissible policies

From the notation considered, $\Gamma$ denotes the set of admissible policies for the $B T$-problem and $\tilde{\Gamma}$ the set of admissible policies for problems $\left\{\mathcal{P}_{k}\right\}$. We have $\Gamma \subset \tilde{\Gamma}$ because a policy that empties the data by the deadline is clearly an admissible policy for the modified problems $\left\{\mathcal{P}_{k}\right\}$ in which case such a policy simply incurs zero terminal energy cost. Thus, for all $r(\cdot) \in \Gamma, x(T)=0$ and the terminal energy cost is zero. This gives for all $k$,

$$
J_{r}^{k}(x, c, t)=J_{r}(x, c, t), \forall r(\cdot) \in \Gamma
$$

where $J_{r}(\cdot)$ above is defined in (5). From Lemma 1 we know that,

$$
J^{k}(x, c, t) \leq J_{r}^{k}(x, c, t), \forall r(\cdot) \in \tilde{\Gamma} \supset \Gamma
$$

Thus from (35) and (36) we have,

$$
J^{k}(x, c, t) \leq J_{r}(x, c, t), \forall r(\cdot) \in \Gamma
$$

Since the above inequality holds for all $k$, taking limits gives,

$$
J(x, c, t) \triangleq \lim _{k \rightarrow \infty} J^{k}(x, c, t) \leq J_{r}(x, c, t), \forall r(\cdot) \in \Gamma
$$

Step 3: Result 2 stated in the theorem

From the theorem statement, we know that $J(x, c, t)$ satisfies (14) and $r^{*}(x, c, t)$ is an admissible policy for the $B T$ problem. Now, using Dynkin's formula, [23], on $J(x, c, t)$ for policy $r^{*}(\cdot)$ we get $\forall \tau, t<\tau<T$,

$$
\begin{aligned}
J(x, c, t) & =E J\left(x_{\tau}, c_{\tau}, \tau\right)-E \int_{t}^{\tau} \mathcal{A}^{r^{*}} J\left(x_{s}, c_{s}, s\right) d s \\
& =E J\left(x_{\tau}, c_{\tau}, \tau\right)+E \int_{t}^{\tau} \frac{g\left(r^{*}\left(x_{s}, c_{s}, s\right)\right)}{c_{s}} d s \\
& \geq E \int_{t}^{\tau} \frac{g\left(r^{*}\left(x_{s}, c_{s}, s\right)\right)}{c_{s}} d s
\end{aligned}
$$

where we have used $x_{s}, c_{s}$ as short-hand notations for $x(s)$ and $c(s)$ respectively. The equality in (40) follows since $r^{*}$ is the minimizing value in (13) which gives $\mathcal{A}^{r^{*}} J(x, c, t)+\frac{1}{c} g\left(r^{*}\right)=$ 0 or equivalently $\frac{1}{c_{s}} g\left(r^{*}\left(x_{s}, c_{s}, s\right)\right)=-\mathcal{A}^{r^{*}} J\left(x_{s}, c_{s}, s\right)$. The inequality in (41) follows since $J(\cdot)$ is non-negative. Since the above holds for all $\tau<T$, taking limits and using the monotone convergence theorem we get,

$$
J(x, c, t) \geq E\left[\int_{t}^{T} \frac{g\left(r^{*}\left(x_{s}^{*}, c_{s}, s\right)\right)}{c_{s}} d s\right]
$$

Combining the above inequality with that in (38) shows that we have equality for policy $r^{*}(x, c, t)$, i.e. $J(x, c, t)=$ $E \int_{t}^{T} \frac{g\left(r^{*}\left(x_{s}, c_{s}, s\right)\right)}{c_{s}} d s$. This completes the proof that $J(x, c, t)$ is the minimum cost function and $r^{*}(\cdot)$ is the optimal policy.

\section{APPENDIX B \\ ProOf OF THEOREM I - BT-problem}

To prove optimality, we check all the conditions required in the verification results of Appendix A as follows. We first consider the relaxed problem $\mathcal{P}_{k}$ and obtain the optimal solution by verifying the conditions in Lemma 1 . Then, we take the limit $k \rightarrow \infty$ and check the conditions required in Theorem V. These limits give us the optimal solution for the $B T$-problem.

Step 1: Optimal solution for the modified problem $\mathcal{P}_{k}$

Let us suppose that the functional form for the optimal rate $r_{k}^{*}(x, c, t)$ is given as,

$$
r_{k}^{*}\left(x, c^{i}, t\right)=\frac{x}{f_{i}^{k}(T-t)}, \quad i=1, \ldots, m
$$

Assuming this functional form we now obtain the minimum cost function $J^{k}(x, c, t)$. To proceed, note that $r_{k}^{*}(\cdot)$ must be the minimizing value of $r$ in (14). Thus, using the first-order 
condition for the minimization (i.e. first derivative with respect to $r$ equal to zero) we get, $\forall(x, c, t) \in(0, B] \times \mathcal{C} \times[0, T)$,

$$
\begin{aligned}
& \frac{\partial}{\partial r}\left(\frac{g(r)}{c^{i}}+\frac{\partial J^{k}\left(x, c^{i}, t\right)}{\partial t}-r \frac{\partial J^{k}\left(x, c^{i}, t\right)}{\partial x}\right. \\
& \left.\quad+\lambda\left(E_{z}\left[J^{k}\left(x, Z\left(c^{i}\right) c^{i}, t\right)\right]-J^{k}\left(x, c^{i}, t\right)\right)\right)\left.\right|_{r_{k}^{*}}=0
\end{aligned}
$$

This gives, $\frac{\partial J^{k}\left(x, c^{i}, t\right)}{\partial x}=\frac{g^{\prime}\left(r_{k}^{*}\left(x, c^{i}, t\right)\right)}{c^{i}}$ and upon integration with the boundary condition $J^{k}\left(0, c^{i}, t\right)=0$, we get,

$$
J^{k}\left(x, c^{i}, t\right)=\frac{x^{n}}{c^{i}\left(f_{i}^{k}(T-t)\right)^{n-1}}, \quad i=1, \ldots, m
$$

In order for the functional forms in (43) and (45) to be the optimal solution we need to satisfy the conditions in Lemma 1.

- First, the boundary condition $J^{k}\left(x, c^{i}, T\right)=h_{k}\left(x, c^{i}\right)=$ $\frac{\left(x / \tau_{k}\right)^{n} \tau_{k}}{c^{i}}$, requires,

$$
f_{i}^{k}(0)=\tau_{k}, \quad \forall i=1, \ldots, m
$$

The other boundary condition $J^{k}\left(0, c^{i}, t\right)=0, \forall i, t$, is already satisfied as can be easily checked.

- Second, $J^{k}(\cdot)$ and $r_{k}^{*}(\cdot)$ must solve the PDE equation in (14) for all values of the system state $(x, c, t) \in$ $\left([0, \infty) \times\left(c_{1}, \ldots, c_{m}\right) \times[0, T)\right)$. That is, we require,

$$
\begin{array}{r}
\frac{g\left(r_{k}^{*}\left(x, c^{i}, t\right)\right)}{c^{i}}+\frac{\partial J^{k}\left(x, c^{i}, t\right)}{\partial t}-r_{k}^{*}\left(x, c^{i}, t\right) \frac{\partial J^{k}\left(x, c^{i}, t\right)}{\partial x} \\
\quad+\lambda\left(E_{z}\left[J^{k}\left(x, Z\left(c^{i}\right) c^{i}, t\right)\right]-J^{k}\left(x, c^{i}, t\right)\right)=0 \text { (47) }
\end{array}
$$

Substituting (43) and (45) in the equation above, simplifying and setting $s=T-t$ gives the following ODE system, $\forall i=1, \ldots, m$,

$$
\left(f_{i}^{k}\right)^{\prime}(s)=1+\frac{\lambda f_{i}^{k}(s)}{n-1}-\frac{\lambda}{n-1} \sum_{j=1}^{m} \frac{p_{i j}}{z_{i j}} \frac{\left(f_{i}^{k}(s)\right)^{n}}{\left(f_{j}^{k}(s)\right)^{n-1}}
$$

Thus, from above we see that for $r_{k}^{*}(\cdot)$ and $J^{k}(\cdot)$, as given in (43) and (45), to satisfy the optimality PDE equation in (14) we require that the functions $\left\{f_{i}^{k}(s)\right\}_{i=1}^{m}$ satisfy the above ODE system with the boundary conditions in (46). The question that remains is whether a set of positive functions exist that solve the ODE system in (48). The following lemma shows that indeed such a set exists and also that these functions are unique.

Lemma 2: (Existence and Uniqueness of the ODE solution in (48)) The ODE system in (48) with the boundary conditions $f_{i}^{k}(0)=\tau_{k}, \forall i, \tau_{k}>0$, has a unique positive solution for $s \in[0, T]$.

Proof: See Appendix C.

Thus, we see that $J^{k}\left(x, c^{i}, t\right)$ as given in (45) solves (14) with the minimizing rate function $r_{k}^{*}\left(x, c^{i}, t\right)$ as given in (43). This rate function is a valid transmission policy as it satisfies all the admissibility requirements for problem $\mathcal{P}_{k}$ (i.e. $r^{*} \geq 0$, $r^{*}=0$ for $x=0, r_{k}^{*}\left(x, c^{i}, t\right)$ is locally Lipschitz continuous in $x$ and continuous in $t$ ). From Lemma 1, it then follows that $J^{k}\left(x, c^{i}, t\right)$ and $r_{k}^{*}\left(x, c^{i}, t\right)$ are the optimal solution for problem $\mathcal{P}_{k}$.

Step 2: Optimal solution for the BT-problem (taking $\lim _{k \rightarrow \infty}$ in the Step 1 results)
Consider the limit $J(x, c, t)=\lim _{k \rightarrow \infty} J^{k}(x, c, t)$. From Theorem $\mathrm{V}$ we know that this limit exists and using (45) we obtain,

$J\left(x, c^{i}, t\right)=\lim _{k \rightarrow \infty} J^{k}\left(x, c^{i}, t\right)=\frac{x^{n}}{c^{i}\left(f_{i}(T-t)\right)^{n-1}}, i=1, \ldots, m$

where we define,

$$
f_{i}(s) \triangleq \lim _{k \rightarrow \infty} f_{i}^{k}(s), \quad s \in[0, T], \forall i
$$

For optimality we now check the conditions required in Theorem V. First, we need to show that $J(\cdot)$ as obtained in (49) satisfies the HJB equation in (14). Substituting the above form of $J\left(x, c^{i}, t\right)$ in (14) and using the first-order condition for the minimization we get, $\frac{\partial J\left(x, c^{i}, t\right)}{\partial x}=\frac{g^{\prime}\left(r^{*}\left(x, c^{i}, t\right)\right)}{c^{i}}$ which gives,

$$
r^{*}\left(x, c^{i}, t\right)=\frac{x}{f_{i}(T-t)}, i=1, \ldots, m
$$

Furthermore, to satisfy the PDE equation we require (see the steps presented in Step 1),

$f_{i}^{\prime}(s)=1+\frac{\lambda f_{i}(s)}{n-1}-\frac{\lambda}{n-1} \sum_{j=1}^{m} \frac{p_{i j}}{z_{i j}} \frac{\left(f_{i}(s)\right)^{n}}{\left(f_{j}(s)\right)^{n-1}}, \forall i=1, \ldots, m$

Thus, equivalently, in order to prove that $J(x, c, t)$ satisfies the HJB equation, we need to show that the functions $\left\{f_{i}(s)\right\}$ as defined in (50) satisfy the above ODE system with the boundary conditions $f_{i}(0)=0$ and $f_{i}^{\prime}(0)=1, \forall i$. These boundary conditions follow by taking the limit $k \rightarrow \infty$ in $f_{i}^{k}(s)$; specifically, $f_{i}^{k}(0)=\tau_{k} \rightarrow 0$ and $\left(f_{i}^{k}\right)^{\prime}(0)=$ $\left(1+\frac{\lambda \tau_{k}}{n-1}-\tau_{k} \sum_{j=1}^{m} \frac{p_{i j}}{z_{i j}}\right) \rightarrow 1$ (Note that as $k \rightarrow \infty, \tau_{k} \downarrow 0$ ). The following lemma shows that this is indeed true and $\left\{f_{i}(s)\right\}$ as defined in (50) satisfy the ODE system in (52) with the above mentioned boundary conditions; furthermore $\left\{f_{i}(s)\right\}$ are also the unique solution of that ODE system.

Lemma 3: The functions $\left\{f_{i}(s)\right\}$ as defined in (50) are the unique solution of the ODE system in (52) with the boundary conditions $f_{i}(0)=0$ and $f_{i}^{\prime}(0)=1, \forall i$.

Proof: See Appendix D.

Finally, we need to check the admissibility of policy $r^{*}(x, c, t)$ as given in (51). To see this, note that the optimal rate $r^{*}$ is non-negative and is zero when $x=0\left(r^{*}(\cdot)\right.$ is locally Lipschitz continuous in $x$ and continuous in $t$ ). The policy $r^{*}(\cdot)$ also satisfies the deadline constraint $x(T)=0$ since the boundary condition, $f_{i}^{\prime}(0)=1, \forall i$, implies that very close to the deadline $T$, the policy behaves as $r^{*}\left(x, c^{i}, t\right)=\frac{x}{T-t}$; thereby emptying the buffer by the deadline.

\section{APPENDIX C}

Proof of Lemma 2 - Existence and Uniqueness of the solution to the $O D E$ in (48)

To ease the notations, let us abstract the ODE system in (48) as follows. Let $x_{i}(s) \triangleq f_{i}^{k}(s), a_{i} \triangleq \frac{\lambda}{n-1}, b_{i j} \triangleq \frac{\lambda p_{i j}}{(n-1) z_{i j}}$, then (48) can be re-written as,

$$
x_{i}^{\prime}(s)=1+a_{i} x_{i}(s)-\sum_{j=1}^{m} b_{i j} \frac{x_{i}(s)^{n}}{x_{j}(s)^{n-1}}, \quad \forall i=1, \ldots, m
$$


where for $p_{i j} \geq 0,\left(\lambda, z_{i j}\right)>0, n>1$, we have, $a_{i}>0$ and $b_{i j} \in[0, \infty)$. Thus, we now have to find a vector of functions $\overline{\mathbf{x}}(s) \triangleq\left(x_{1}(s), \ldots, x_{m}(s)\right)$ such that each $x_{i}(s)$ satisfies the equation in (53) with the initial condition $\overline{\mathbf{x}}(0)=\left(\tau_{k}, \ldots, \tau_{k}\right)$ (since $f_{i}^{k}(0)=\tau_{k}, \forall i$ ).

Let us define $G_{i}(\overline{\mathbf{x}}(s)) \triangleq 1+a_{i} x_{i}(s)-\sum_{j=1}^{m} b_{i j} \frac{x_{i}(s)^{n}}{x_{j}(s)^{n-1}}$, then, in a very compact form we get,

$$
\overline{\mathbf{x}}^{\prime}(s)=\overline{\mathbf{G}}(\overline{\mathbf{x}}(s))
$$

where $\overline{\mathbf{x}}^{\prime}(s)$ is the column vector $\left(x_{1}^{\prime}(s), \ldots, x_{m}^{\prime}(s)\right)$ and $\overline{\mathbf{G}}(\cdot)$ is the column vector $\left(G_{1}(\cdot), \ldots, G_{m}(\cdot)\right)$. Now consider the open positive orthant and denote it as $\mathcal{U}$, thus, $\mathcal{U}=\left(x_{1}>0, \ldots, x_{m}>0\right)$. For $\overline{\mathbf{x}} \in \mathcal{U}$, each $G_{i}(\overline{\mathbf{x}})$ is a continuously differentiable function. Hence, $\overline{\mathbf{G}}(\overline{\mathbf{x}})$ is continuously differentiable which means that it is locally Lipschitz continuous in $\overline{\mathrm{x}}$ over the set $\mathcal{U}$. Therefore, starting with $\overline{\mathbf{x}}(0)=\left(\tau_{k}, \ldots, \tau_{k}\right) \in \mathcal{U}$, the ODE in (54) has a unique local solution $\overline{\mathbf{x}}(s)$ that lies in $\mathcal{U}$ [26]. The only question now remains is whether the local solution leaves the open positive orthant, i.e. whether $\overline{\mathbf{x}}(s) \notin \mathcal{U}$ for some finite $s>0$. And the answer is no; the local solution remains inside $\mathcal{U}$, which then proves the claim that the ODE in (54) has a unique positive solution for all $s>0$. To prove the last requirement that $\overline{\mathbf{x}}(s) \in \mathcal{U}, \forall s>0$, we proceed as follows.

First, since $\overline{\mathbf{G}}(\overline{\mathbf{x}})$ is locally Lipschitz continuous in $\overline{\mathbf{x}}$, a unique local solution that lies in $\mathcal{U}$ exists for the ODE in (54). Suppose now that $0<s_{0}<\infty$ is the first instant at which for some $i$, we have $x_{i}\left(s_{0}\right)=0$ or $x_{i}\left(s_{0}\right)=\infty$, i.e. $s_{0}$ is the first instant at which $\overline{\mathbf{x}}(s)$ leaves the positive orthant $\mathcal{U}$. Over the interval $s \in\left[0, s_{0}\right)$ we have,

$$
\begin{aligned}
x_{i}^{\prime}(s) & =1+a_{i} x_{i}(s)-\sum_{j=1}^{m} b_{i j} \frac{x_{i}(s)^{n}}{x_{j}(s)^{n-1}} \\
& \leq 1+a_{i} x_{i}(s)
\end{aligned}
$$

From (56) above we get,

$$
x_{i}(s) \leq \frac{\left(1+a_{i} \tau_{k}\right) e^{a_{i} s}-1}{a_{i}}
$$

Thus, each $x_{i}(s)$ is bounded above by an exponential function that goes to infinity only when $s \rightarrow \infty$.

We now compute the lower bound on the functions $\left\{x_{i}(s)\right\}$. Let $x_{l}(s)$ take the smallest value among $\left\{x_{i}(s)\right\}$ over an interval $\left[0, s_{1}\right], s_{1} \leq s_{0}$; this is true since $x_{i}(s)$ are continuous functions and if a certain function takes the smallest value, it will remain the minimum over some interval. We then have, $x_{l}(s) / x_{j}(s) \leq 1, \forall j=1, \ldots, m$ over $s \in\left[0, s_{1}\right]$. This gives,

$$
\begin{aligned}
x_{l}^{\prime}(s) & =1+a_{l} x_{l}(s)-\sum_{j=1}^{m} b_{l j} \frac{x_{l}(s)^{n}}{x_{j}(s)^{n-1}} \\
& \geq-\sum_{j=1}^{m} b_{l j} \frac{x_{l}(s)^{n}}{x_{j}(s)^{n-1}}=-x_{l}(s) \sum_{j=1}^{m} b_{l j}\left(\frac{x_{l}(s)}{x_{j}(s)}\right)^{n-1} \\
& \geq-x_{l}(s) \sum_{j=1}^{m} b_{l j}, \quad\left(\text { since } x_{l}(s) / x_{j}(s) \leq 1, \forall j\right) \\
& =-c_{l} x_{l}(s), \quad\left(\text { taking } c_{l}=\sum_{j=1}^{m} b_{l j}\right)
\end{aligned}
$$

From (61) above we get,

$$
x_{l}(s) \geq \tau_{k} e^{-s c_{l}}
$$

Thus, $x_{l}(s)$ is bounded below by an exponential function that goes to zero only when $s \rightarrow \infty$. Using a recursive argument starting with $s=s_{1}$ and following the new minimum function, it follows that over the interval $\left[0, s_{0}\right)$ all $x_{i}(s)$ are lower bounded by $\tau_{k} e^{-s c_{\max }}$, where $c_{\max }=\max _{l=1, \ldots, m} c_{l}$.

From the arguments above we therefore deduce that the unique local solution, $\overline{\mathbf{x}}(s)$, is upper and lower bounded by two respective positive exponential functions. Hence, the local solution never leaves the set $\mathcal{U}$. Thus, by contradiction $s_{0}$ cannot be finite and it then follows that the ODE in (54) has a unique positive global solution, $\overline{\mathbf{x}}(s)$, for all $s>0$, i.e. we have a unique $\overline{\mathbf{x}}(s) \in \mathcal{U}, \forall s>0$ that satisfies (54) with $\overline{\mathbf{x}}(0)=\left(\tau_{k}, \ldots, \tau_{k}\right)$.

\section{APPENDIX D}

Proof of Lemma 3 - Functions $\left\{f_{i}(s)\right\}$ are the unique solution of the ODE system in (52)

We know from Lemma 2 that $f_{i}^{k}(s)$ is a continuously differentiable function, hence, $\left(f_{i}^{k}\right)^{\prime}(s)$ exists for all $s \in[0, T]$ and from (48) it is given as,

$\left(f_{i}^{k}\right)^{\prime}(s)=1+\frac{\lambda f_{i}^{k}(s)}{n-1}-\frac{\lambda}{n-1} \sum_{j=1}^{m} \frac{p_{i j}}{z_{i j}} \frac{\left(f_{i}^{k}(s)\right)^{n}}{\left(f_{j}^{k}(s)\right)^{n-1}}, i=1, . ., m$

Take the limit $k \rightarrow \infty$ in the above equation and denote this point-wise limit as $h_{i}(s)$, i.e. $h_{i}(s) \triangleq \lim _{k \rightarrow \infty}\left(f_{i}^{k}\right)^{\prime}(s)$. The limit exists since $f_{i}^{k}(s)$ is pointwise convergent for all $i$ (see Step 2 of Appendix B). Thus, we get,

$$
\begin{aligned}
h_{i}(s) & =1+\frac{\lambda f_{i}(s)}{n-1}-\frac{\lambda}{n-1} \sum_{j=1}^{m} \frac{p_{i j}}{z_{i j}} \frac{\left(f_{i}(s)\right)^{n}}{\left(f_{j}(s)\right)^{n-1}}, s \in(0, T)(64) \\
& =1, \quad s=0
\end{aligned}
$$

To prove the lemma, we need to show that $f_{i}(s)$ as defined in (50) satisfies $f_{i}^{\prime}(s)=h_{i}(s)$. To do this, we use the following result [27] (Thm. 7.17, pg. 152).

Lemma 4: [27] Suppose $\left\{f_{n}\right\}$ is a sequence of functions, differentiable on $[a, b]$ and such that $\left\{f_{n}\left(x_{0}\right)\right\}$ converges for some point $x_{0}$ on $[a, b]$. If $\left\{f_{n}^{\prime}\right\}$ converges uniformly on $[a, b]$, then $\left\{f_{n}\right\}$ converges uniformly on $[a, b]$, to a function $f$, and $f^{\prime}(x)=\lim _{n \rightarrow \infty} f_{n}^{\prime}(x),(a \leq x \leq b)$.

For our case, for all $i,\left\{f_{i}^{k}(s)\right\}_{k=1}^{\infty}$ forms a sequence of differentiable functions on $[0, T]$ and $f_{i}^{k}(s)$ converges pointwise to $f_{i}(s)$. We show in Lemma 5 below that $\left(f_{i}^{k}\right)^{\prime}(s)$ has a uniformly convergent subsequence on $[0, T]$. Considering this subsequence, combined with Lemma 4 above (for our case, the sequence $\left\{f_{n}^{\prime}\right\}$ in Lemma 4 is the uniformly convergent subsequence $\left\{\left(f_{i}^{k}\right)^{\prime}(s)\right\}$ and the limit function $f$ is $f_{i}(s)$ ), we obtain, $f_{i}^{\prime}(s)=h_{i}(s)=1+\frac{\lambda f_{i}(s)}{n-1}-\frac{\lambda}{n-1} \sum_{j=1}^{m} \frac{p_{i j}}{z_{i j}} \frac{\left(f_{i}(s)\right)^{n}}{\left(f_{j}(s)\right)^{n-1}}$ (from (64)). Thus, this proves that $f_{i}(s)$ is a solution of the ODE as given in (52) with $f_{i}(0)=0$ and $f_{i}^{\prime}(0)=1$.

Lemma 5: (Uniform convergence of $\left(f_{i}^{k}\right)^{\prime}(s)$ ) The functions $\left\{\left(f_{i}^{k}\right)^{\prime}(s)\right\}_{k=1}^{\infty}$ have a uniformly convergent subsequence on $s \in[0, T]$ for all $i$. 
Proof: The proof is omitted here for brevity and can be found in [3].

We now prove uniqueness using a contradiction argument. Suppose that the solution is not unique and let $\overline{\mathbf{f}}(s)=$ $\left(f_{1}(s), \ldots, f_{m}(s)\right)$ and $\overline{\mathbf{y}}(s)=\left(y_{1}(s), \ldots, y_{m}(s)\right)$ be two solutions with $y_{i}(0)=0, y_{i}^{\prime}(0)=1, f_{i}(0)=0, f_{i}^{\prime}(0)=$ $1, \forall i=1, \ldots, m$. We first show that if we look at $s$ close to 0 , the two solutions $\overline{\mathbf{y}}(s)$ and $\overline{\mathbf{f}}(s)$ are in the positive orthant and close to each other. Start at $s=0$ and consider $\epsilon>0$, then by the mean value theorem [27] we have, $y_{i}(\epsilon)=\epsilon y_{i}^{\prime}(\eta)$ with $\eta \in(0, \epsilon)$. By the continuity of the derivative, we further have $y_{i}(\epsilon)=\epsilon\left(y_{i}^{\prime}(0)+\gamma_{i}(\epsilon)\right)=\epsilon\left(1+\gamma_{i}(\epsilon)\right)$, where $\gamma_{i}(\epsilon) \stackrel{\epsilon \rightarrow 0}{\longrightarrow} 0$ and this holds for all $i$. Thus for $\epsilon$ small enough we must have $\overline{\mathbf{y}}(\epsilon)>0$; in other words there exists a $\tilde{\epsilon}$ such that for all $0<\epsilon<\tilde{\epsilon}$ the solution $\overline{\mathbf{y}}(\epsilon)$ is in the positive orthant. Similarly, since $\overline{\mathbf{f}}(s)=\left(f_{1}(s), \ldots, f_{m}(s)\right)$ is also a solution, the above set of arguments hold for it as well and we have, $\overline{\mathbf{f}}(\epsilon)>0$. From above we also see that $\|\overline{\mathbf{y}}(\epsilon)\|<\gamma_{y}(\epsilon)$, where $\gamma_{y}(\epsilon) \stackrel{\epsilon \rightarrow 0}{\longrightarrow} 0$ and a similar inequality holds for $\overline{\mathbf{f}}(\epsilon)$ as well. Thus, $\|\overline{\mathbf{y}}(\epsilon)-\overline{\mathbf{f}}(\epsilon)\|<\|\overline{\mathbf{y}}(\epsilon)\|+\|\overline{\mathbf{f}}(\epsilon)\|<\left(\gamma_{y}(\epsilon)+\gamma_{f}(\epsilon)\right)$.

Now, pick $\epsilon \in(0, \tilde{\epsilon})$ and consider the two solutions of the ODE over time $s \in[\epsilon, T]$ starting from the initial state $\overline{\mathbf{y}}(\epsilon)$ and $\overline{\mathbf{f}}(\epsilon)$ respectively. Following the proof of Lemma 2 (Appendix C), we see that starting from an initial state in the positive orthant, the ODE has a unique solution that lies in the positive orthant. Furthermore, from [26], the solution is continuous with respect to the initial conditions. Thus, this implies that starting with close enough initial conditions the two solutions $\overline{\mathbf{y}}(s)$ and $\overline{\mathbf{f}}(s)$ must be close enough for all $s \in[\epsilon, T]$. Mathematically, for any $\zeta>0$, there exists an $\epsilon \in(0, \tilde{\epsilon})$ such that $\max _{s \in[\epsilon, T]}\|\overline{\mathbf{y}}(s)-\overline{\mathbf{f}}(s)\|<\zeta$. By taking $\zeta$ going to zero, we see that $\overline{\mathbf{y}}(s)$ and $\overline{\mathbf{f}}(s)$ cannot be distinct over $s \in[0, T]$ and this completes the proof.

\section{APPENDIX E}

\section{Proof of Theorem II - Constant Drift Channel, Monomial Case}

The proof for this result is identical to that of Theorem I but now we can evaluate the $\left\{f_{i}(s)\right\}$ functions in closed form. To see this, start with problem $\mathcal{P}_{k}$ and suppose that for all channel states the $f_{i}^{k}(s)$ function is the same, i.e. $f_{i}^{k}(s)=f^{k}(s)$. The ordinary differential for $f^{k}(s)$ then becomes,

$$
\begin{aligned}
\left(f^{k}\right)^{\prime}(s) & =1+\frac{\lambda f^{k}(s)}{n-1}-\frac{\lambda}{n-1} f^{k}(s)\left(\sum_{j} \frac{p_{i j}}{z_{i j}}\right) \\
& =1-\frac{\lambda f^{k}(s)}{n-1}(\beta-1)
\end{aligned}
$$

where $\sum_{j} \frac{p_{i j}}{z_{i j}}=E\left[1 / Z\left(c^{i}\right)\right]=\beta, \forall i$, by the constant drift channel assumption. The solution to the above ODE with boundary condition $f^{k}(0)=\tau_{k}$ is given as,

$$
\begin{aligned}
f^{k}(s)=\frac{(n-1)}{\lambda(\beta-1)} & \left(1-\exp \left(-\frac{\lambda(\beta-1) s}{n-1}\right)\right) \\
& +\tau_{k} \exp \left(-\frac{\lambda(\beta-1) s}{n-1}\right), s \geq 0
\end{aligned}
$$

From Lemma 2 the above function is the unique solution of the ODE in (67) and it can be easily checked that the functional forms $r_{k}^{*}(x, c, t)=\frac{x}{f^{k}(T-t)}$ and $J^{k}(x, c, t)=\frac{x^{n}}{c\left(f^{k}(T-t)\right)^{n-1}}$ satisfy the conditions in Lemma 1 . To obtain the solution for the $B T$-problem, we take the limit $(k \rightarrow \infty)$ which gives the optimal solution in (21) and (22) with $f(s) \triangleq \lim _{k \rightarrow \infty} f^{k}(s)=$ $\frac{(n-1)}{\lambda(\beta-1)}\left(1-\exp \left(-\frac{\lambda(\beta-1) s}{n-1}\right)\right)$.

\section{APPENDIX F}

\section{Proof of Theorem III - Constant Drift Channel, Exponential Case}

A direct non-constructive proof for showing optimality is to plug the functional forms given in the theorem statement into the PDE equation in (14) and check if it satisfies the equation. However, such a proof would not reveal how the particular functional form can be obtained. To present a constructive proof, we utilize discrete dynamic programming and proceed as follows. From the steps in Appendix A, we first solve for the optimal functions, $\left\{J^{k}(x, c, t), r_{k}^{*}(x, c, t)\right\}$, of the relaxed problem $\mathcal{P}_{k}$, take the limit $\tau_{k} \downarrow 0$ and verify the conditions of Theorem V. Now, to solve problem $\mathcal{P}_{k}$, we consider a discrete approximation of the time interval $[0, T]$ with step size $d t$. Using dynamic programming (DP), we obtain the functional form of the optimal policy and the minimum cost function and take the limit $d t \rightarrow 0$. Thus, there are two limiting operations involved, first $d t \rightarrow 0$ to solve for the optimal functions for problem $\mathcal{P}_{k}$ and then $\tau_{k} \downarrow 0$ to solve for the optimal functions for the $B T$-problem. We treat the two cases, $\beta \geq 1$ and $\beta<1$ separately.

Case 1: $\beta \geq 1$. Consider a discrete approximation of time with step size $d t>0$. Starting at time $T$ and recursing backwards, let $[T-j d t, T-(j-1) d t], j \geq 1$ denote the $j^{\text {th }}$ stage and $V_{j}(x, c)$ the corresponding cost-to-go function starting with $x$ amounts of data and channel state $c$. Denote the $j^{\text {th }}$ stage optimal transmission rate as $r_{j}(x, c)$. Let $V_{0}$ denote the terminal energy cost over $\left[T, T+\tau_{k}\right]$, then, $V_{0}(x, c)=$ $h_{k}(x, c)=\frac{\left(\alpha^{x / \tau_{k}}-1\right)}{c} \tau_{k}$. The first step DP recursion is,

$$
\begin{gathered}
V_{1}(x, c)=\min _{0 \leq r \leq x / d t}\left\{\frac{\left(\alpha^{r}-1\right) d t}{c}+(1-\lambda d t) V_{0}(x-r d t, c)\right. \\
\left.+\lambda d t E_{z}\left(V_{0}(x-r d t, Z c)\right)\right\}
\end{gathered}
$$

The constraint $0 \leq r \leq x / d t$ follows from the non-negativity of the rate and the buffer state respectively. Substituting $V_{0}(\cdot)$ and using standard lagrangian techniques, it is easy to show that the above minimization has the following solution. Let $\rho=1+\lambda d t(\beta-1),(\rho \geq 1$, since $\beta \geq 1)$,

$$
\begin{aligned}
& r_{1}(x, c)= \begin{cases}\frac{x}{\tau_{k}+d t}+\frac{\tau_{k} \ln \rho}{\left(\tau_{k}+d t\right) \ln \alpha}, & x \geq \frac{d t \ln \rho}{\ln \alpha} \\
\frac{x}{d t}, & 0 \leq x<\frac{d t \ln \rho}{\ln \alpha}\end{cases} \\
& V_{1}(x, c)= \begin{cases}\frac{\tau_{k}+d t}{c} \alpha^{r_{1}(x, c)-\frac{\left(d t+\rho \tau_{k}\right)}{c},} & x \geq \frac{d t \ln \rho}{\ln \alpha} \\
\frac{d t}{c}\left(\alpha^{r_{1}(x, c)}-1\right), & 0 \leq x<\frac{d t \ln (}{\ln \alpha}\end{cases}
\end{aligned}
$$

Following the DP recursion for the next stage, we get,

$$
\begin{aligned}
V_{2}(x, c)=\min _{0 \leq r \leq x / d t} & \left\{\frac{\left(\alpha^{r}-1\right) d t}{c}+(1-\lambda d t) V_{1}(x-r d t, c)\right. \\
& \left.+\lambda d t E_{z}\left(V_{1}(x-r d t, Z c)\right)\right\}
\end{aligned}
$$


Now, to solve the above minimization, first assume $x-r d t \geq$ $\frac{d t \ln \rho}{\ln \alpha}$. Substituting the corresponding form of $V_{1}(\cdot)$ into (72) and solving the minimization by standard differentiation, the optimal rate can be obtained as $r=\frac{x}{\tau_{k}+2 d t}+\frac{\left(2 \tau_{k}+d t\right) \ln \rho}{\left(\tau_{k}+2 d t\right) \ln \alpha}$. With this optimal $r$, substituting in $x-r d t \geq \frac{d t \ln \rho}{\ln \alpha}$, we get the threshold, $x \geq \frac{3 d t \ln \rho}{\ln \alpha}$. Note that for the above threshold $r d t \leq x$, thus, buffer non-negativity constraint is also satisfied. Next, assume $x-r d t<\frac{d t \ln \rho}{\ln \alpha}$, and substitute the relevant form of $V_{1}(\cdot)$ into (72). Proceeding as before, we get, $r=$ $\frac{x}{2 d t}+\frac{\ln \rho}{2 \ln \alpha}$. Using this $r$ in $x-r d t<\frac{d t \ln \rho}{\ln \alpha}$ and the buffer non-negativity constraint $x-r d t \geq 0$ we get the threshold on $x$ as, $\frac{d t \ln \rho}{\ln \alpha} \leq x \leq \frac{3 d t \ln \rho}{\ln \alpha}$. Finally, for $x<\frac{d t \ln \rho}{\ln \alpha}$, all the data is drained in the second stage and the rate is $r=\frac{x}{d t}$. Thus, the solution of the minimization in (72) is,

$r_{2}(x, c)=\left\{\begin{array}{l}\frac{x}{\tau_{k}+2 d t}+\frac{\left(2 \tau_{k}+d t\right) \ln \rho}{\left(\tau_{k}+2 d t\right) \ln \alpha}, \quad x \geq \frac{3 d t \ln \rho}{\ln \alpha} \\ \frac{x}{2 d t}+\frac{\ln \rho}{2 \ln \alpha}, \quad \frac{d t \ln \rho}{\ln \alpha} \leq x<\frac{3 d t \ln \rho}{\ln \alpha} \\ \frac{x}{d t}, \quad x<\frac{d t \ln \rho}{\ln \alpha}\end{array}\right.$
$V_{2}(x, c)=\left\{\begin{array}{l}\frac{\tau_{k}+2 d t}{c} \alpha^{r_{2}(x, c)}-\frac{\left(d t+\rho d t+\rho^{2} \tau_{k}\right)}{c}, x \geq \frac{3 d t \ln \rho}{\ln \alpha} \\ \frac{2 d t}{c} \alpha^{r_{2}(x, c)}-\frac{(d t+\rho d t)}{c}, \frac{d t \ln \rho}{\ln \alpha} \leq x<\frac{3 d t \ln \rho}{\ln \alpha} \\ \frac{d t}{c}\left(\alpha^{r_{2}(x, c)}-1\right), x<\frac{d t \ln \rho}{\ln \alpha}\end{array}\right.$

Continuing the DP recursion, the solution for the $j^{\text {th }}$ stage is,

$r_{j}(x, c)=\left\{\begin{array}{l}\frac{x}{\tau_{k}+j d t}+\frac{\left(j \tau_{k}+\frac{j(j-1)}{2} d t\right) \ln \rho}{\left(\tau_{k}+j d t\right) \ln \alpha}, x \geq \frac{j(j+1) d t \ln \rho}{2 \ln \alpha} \\ \frac{x}{2 \ln \alpha}, \\ \frac{(j-i+1) d t}{j-i)(j-i+1) d t \ln \rho} \leq \frac{(j-i) \ln \rho}{2 \ln \alpha} \leq x<\frac{(j-i+1)(j-i+2) d t \ln \rho}{2 \ln \alpha}\end{array}\right.$

$V_{j}(x, c)=\left\{\begin{array}{l}\frac{\tau_{k}+j d t}{c} \alpha^{r_{2}(x, c)}-\frac{\left(1+\rho+\ldots+\rho^{j-1}\right) d t+\rho^{j} \tau_{k}}{c}, \\ x \geq \frac{j(j+1) d t \ln \rho}{2 \ln \alpha} \\ \frac{(j-i+1) d t}{c} \alpha^{r_{2}(x, c)}-\frac{\left(1+\rho+\ldots+\rho^{j-i}\right) d t}{c}, \\ \frac{(j-i)(j-i+1) d t \ln \rho}{2 \ln \alpha} \leq x<\frac{(j-i+1)(j-i+2) d t \ln \rho}{2 \ln \alpha}\end{array}\right.$

where $i=1, \ldots, j$. Now, take the limits, $d t \rightarrow 0, j d t \rightarrow$ $(T-t)$. Under this limiting operation, we have $j \ln \rho \rightarrow \lambda(\beta-$ 1) $(T-t)$. Applying these limits we get (let, $\zeta=\lambda(\beta-1)$ ),

$r_{k}^{*}(x, c, t)=\left\{\begin{array}{l}\sqrt{\frac{2 x \zeta}{\ln \alpha}}, 0 \leq x<\frac{\zeta(T-t)^{2}}{2 \ln \alpha} \\ \frac{x}{\tau_{k}+T-t}+\frac{\zeta(T-t)\left(\tau_{k}+\frac{T-t}{2}\right)}{\left(\tau_{k}+T-t\right) \ln \alpha}, x \geq \frac{\zeta(T-t)^{2}}{2 \ln \alpha}\end{array}\right.$

$J^{k}(x, c, t)=\left\{\begin{array}{l}\frac{1}{c}\left(\sqrt{\frac{2 x \ln \alpha}{\zeta}} e^{\sqrt{2 x \zeta \ln \alpha}}+\frac{1-e^{\sqrt{2 x \zeta \ln \alpha}}}{\zeta}\right) \\ 0 \leq x<\frac{\zeta(T-t)^{2}}{2 \ln \alpha} \\ \frac{1}{c}\left(T-t+\tau_{k}\right) \alpha^{r_{k}^{*}(x, c, t)}-\frac{\left(1+\zeta \tau_{k}\right) e^{\zeta(T-t)}-1}{c \zeta} \\ x \geq \frac{\zeta(T-t)^{2}}{2 \ln \alpha}\end{array}\right.$

The function $J^{k}(x, c, t)$ given in (74) is continuously differentiable, satisfies the HJB equation in (14) and the boundary conditions for problem $\mathcal{P}_{k}$. The policy $r_{k}^{*}(x, c, t)$ is admissible and is the minimizing $r$ for the HJB equation. Thus, by Lemma 1, (73) and (74) form the optimal solution for $\mathcal{P}_{k}$. To obtain $J(x, c, t)$ take the limit $\tau_{k} \downarrow 0$ in (74). This gives,

$$
J(x, c, t)=\left\{\begin{array}{l}
\frac{1}{c}\left(\sqrt{\frac{2 x \ln \alpha}{\zeta}} e^{\sqrt{2 x \zeta \ln \alpha}}+\frac{1-e^{\sqrt{2 x \zeta \ln \alpha}}}{\zeta}\right) \\
0 \leq x<\frac{\zeta(T-t)^{2}}{2 \ln \alpha} \\
\frac{1}{c}\left((T-t) \alpha^{\frac{x}{T-t}}+\frac{\zeta(T-t)}{2 \ln \alpha}-\frac{e^{\zeta(T-t)}-1}{\zeta}\right) \\
x \geq \frac{\zeta(T-t)^{2}}{2 \ln \alpha}
\end{array}\right.
$$

Taking limits in (73) gives $r^{*}(\cdot)$ as in (23). To check optimality, we need to verify the conditions of Theorem $\mathrm{V}$. It is easy to check that $J(x, c, t)$ in (75) satisfies the HJB equation with $r^{*}(\cdot)$ the minimizing value. Policy $r^{*}(\cdot)$, satisfies the admissibility criteria including the deadline constraint, since, the rate $r^{*}(x, c, t)>\frac{x}{T-t}, \forall x>0, t<T$.

Case 2: $\beta<1$. The result follows using the same methodology as in the previous case and is omitted here for brevity. The function $J(x, c, t)$ in this case is (let, $\eta=\lambda(1-\beta)$ ),

$$
J(x, c, t)=\left\{\begin{array}{l}
\frac{e^{-\eta(T-t)}}{c}\left(\sqrt{\frac{2 x \ln \alpha}{\eta}} e^{\sqrt{2 x \eta \ln \alpha}}+\frac{1-e^{\sqrt{2 x \eta \ln \alpha}}}{\eta}\right), \\
0 \leq x<\frac{\eta(T-t)^{2}}{2 \ln \alpha} \\
\frac{1}{c}\left((T-t) \alpha^{\frac{x}{T-t}-\frac{\eta(T-t)}{2 \ln \alpha}}+\frac{e^{-\eta(T-t)}-1}{\eta}\right), \\
x \geq \frac{\eta(T-t)^{2}}{2 \ln \alpha}
\end{array}\right.
$$

\section{APPENDIX G \\ Proof of Theorem IV - Variable Deadlines Setup}

For brevity, we only present the proof for the two packet case $(M=2)$ as it easily helps elucidate the steps involved; the complete proof for arbitrary $M$ can be found in [3].

Two Packet Case: The proof outline is as follows. We start with the functional form for $r^{*}(D, c, t)$ as given in (31), obtain the minimum cost function $J(D, c, t)$ and check that these satisfy the PDE equation in (28). While this simply constitutes a check that the HJB equation is satisfied, to finally complete the optimality proof, we consider a sequence of relaxed problems $\left\{\mathcal{P}_{k}\right\}$ along similar lines as done in Appendix A and then take the appropriate limits. We begin first with the verification that the given rate functional satisfies the HJB equation.

Step 1 - Verification of the HJB Equation: Start with rate function in (31) and consider first the state space $(D, c, t) \in$ $\left[B_{1}, B_{2}\right] \times \mathcal{C} \times\left[T_{1}, T_{2}\right)-$ that is, we are looking at time $t \geq$ $T_{1}$ and all admissible $D$ values over this time. Starting from $(D, c, t)$ in this state space, clearly, the problem is identical to the $B T$-problem with $B=\left(B_{2}-D\right)$ and $T=\left(T_{2}-t\right)$. From (21), the optimal rate function must be $r^{*}(D, c, t)=\frac{B_{2}-D}{f\left(T_{2}-t\right)}$. In conformation, the rate function in (31) over this state space also reduces to the same form. Thus, over this state space, (31) is trivially the optimal policy.

Next consider the state space $(D, c, t) \in\left[0, B_{2}\right] \times \mathcal{C} \times\left[0, T_{1}\right)$; thus now we are considering $0 \leq t<T_{1}$ and all admissible $D$ values over this time which are $\left[0, B_{2}\right]$. Fix a value of $t$ and $c$, then, as a function of $D$ the rate $r^{*}(\cdot)$ in (31) has the following two possibilities.

Case 1: Suppose $\frac{B_{2}}{f\left(T_{2}-t\right)}>\frac{B_{1}}{f\left(T_{1}-t\right)}$. For a fixed $t$, we see that both $\frac{B_{1}-D}{f\left(T_{1}-t\right)}$ and $\frac{B_{2}-D}{f\left(T_{2}-t\right)}$ are linear in $D$. Figure 11(a) gives a schematic picture of the two curves and from the figure it is clear that since $B_{2}>B_{1}$, the two curves do not intersect over $D \in\left[0, B_{1}\right]$. Thus, in this case the maximizing function for all $D \in\left[0, B_{2}\right]$ is $\frac{B_{2}-D}{f\left(T_{2}-t\right)}$ and so, $r^{*}(D, c, t)=\frac{B_{2}-D}{f\left(T_{2}-t\right)}$.

Case 2: Suppose $\frac{B_{2}}{f\left(T_{2}-t\right)} \leq \frac{B_{1}}{f\left(T_{1}-t\right)}$. In this case, the two functions $\frac{B_{1}-D}{f\left(T_{1}-t\right)}$ and $\frac{B_{2}-D}{f\left(T_{2}-t\right)}$ are plotted in Figure 11(b). From the figure it is clear that since $B_{1}<B_{2}$ the two curves must intersect at some $\tilde{B} \in\left[0, B_{1}\right]$ which satisfies $\frac{B_{1}-\tilde{B}}{f\left(T_{1}-t\right)}=$ 


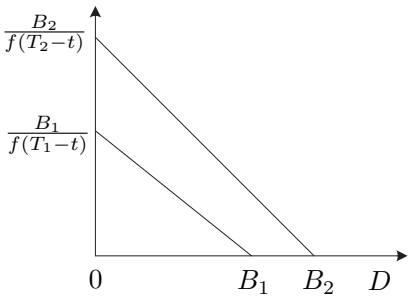

(a)

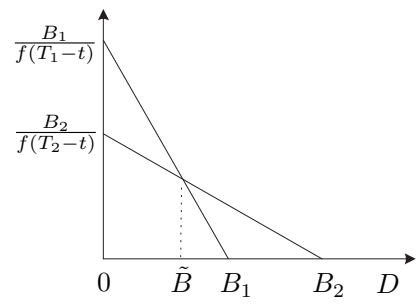

(b)
Fig. 11. Proof of Theorem IV for the two packet case, (a) case $\frac{B_{2}}{f\left(T_{2}-t\right)}>$ $\frac{B_{1}}{f\left(T_{1}-t\right)}$ and (b) case $\frac{B_{2}}{f\left(T_{2}-t\right)} \leq \frac{B_{1}}{f\left(T_{1}-t\right)}$.

$\frac{B_{2}-\tilde{B}}{f\left(T_{2}-t\right)}$. Thus, in this case we get $r^{*}(D, c, t)=\frac{B_{1}-D}{f\left(T_{1}-t\right)}$ for $D \in[0, \tilde{B}]$ and $r^{*}(D, c, t)=\frac{B_{2}-D}{f\left(T_{2}-t\right)}$ for $D \in\left[\tilde{B}, B_{2}\right]$. Define,

$$
\tilde{B}(t) \triangleq \begin{cases}0, & \text { if } \frac{B_{1}}{f\left(T_{1}-t\right)}<\frac{B_{2}}{f\left(T_{2}-t\right)} \\ \frac{B_{1}}{\frac{f\left(T_{1}-t\right)}{1}-\frac{B_{2}}{f\left(T_{2}-t\right)}}, \frac{1}{f\left(T_{2}-t\right)}, & \text { otherwise }\end{cases}
$$

Using (76), $r^{*}(\cdot)$ can be written in a more compact form as,

$$
r^{*}(D, c, t)= \begin{cases}\frac{B_{2}-D}{f\left(T_{2}-t\right)}, & \tilde{B}(t) \leq D \leq B_{2} \\ \frac{B_{1}-D}{f\left(T_{1}-t\right)}, & 0 \leq D<\tilde{B}(t)\end{cases}
$$

The above compact form covers both cases 1 and 2 above - for the first case $\tilde{B}(t)=0$ and for the second case we get $\tilde{B}(t)$ as required. Note that for the constant drift channel, since the function $f(\cdot)$ is the same for all the channel states, the intersection point $\tilde{B}(\cdot)$ as defined in (76) depends only on time $t$ and not on the channel state.

In order for the HJB equation to be satisfied, the rate function $r^{*}(D, c, t)$ above must be the minimizing value in (28). Using the first-order condition for the minimization then gives $\frac{\partial J(D, c, t)}{\partial D}=-\frac{g^{\prime}\left(r^{*}(D, c, t)\right)}{c}$ (note $g(r)=r^{n}$ ); integrating with respect to $D$ with the boundary condition $J\left(B_{2}, c, t\right)=0$, we obtain,

$$
J(D, c, t)=\left\{\begin{array}{l}
\frac{\left(B_{2}-D\right)^{n}}{c\left(f\left(T_{2}-t\right)\right)^{n-1}}, \quad \tilde{B}(t) \leq D \leq B_{2} \\
\frac{\left(B_{1}-D\right)^{n}}{c\left(f\left(T_{1}-t\right)\right)^{n-1}}+\frac{\left(B_{2}-\tilde{B}(t)\right)^{n}}{c\left(f\left(T_{2}-t\right)\right)^{n-1}} \\
-\frac{\left(B_{1}-\tilde{B}(t)\right)^{n}}{c\left(f\left(T_{1}-t\right)\right)^{n-1}}, \quad 0 \leq D<\tilde{B}(t)
\end{array}\right.
$$

To finally verify that the HJB equation is satisfied, we now only need to check that $r^{*}(D, c, t)$ and $J(D, c, t)$ as given in (77) and (78) respectively, satisfy the following PDE,

$$
\begin{aligned}
& \left\{\frac{g\left(r^{*}(D, c, t)\right)}{c}+\frac{\partial J(D, c, t)}{\partial t}+r^{*}(D, c, t) \frac{\partial J(D, c, t)}{\partial D}\right. \\
& \left.+\lambda\left(E_{z}[J(D, Z(c) c, t)]-J(D, c, t)\right)\right\}=0
\end{aligned}
$$

Consider first $D \in\left[\tilde{B}(t), B_{2}\right]$, then, from (78) we have $J(D, c, t)=\frac{\left(B_{2}-D\right)^{n}}{c\left(f\left(T_{2}-t\right)\right)^{n-1}}$ and from (77) we have $r^{*}(D, c, t)=\frac{B_{2}-D}{f\left(T_{2}-t\right)}$. Substituting in the left hand side (LHS) of (79) gives (let $s=T_{2}-t$ ),

$$
\begin{aligned}
\text { LHS } & =\frac{(n-1)\left(B_{2}-D\right)^{n}}{c(f(s))^{n}}\left(f^{\prime}(s)-1+\frac{\lambda(\beta-1)}{n-1} f(s)\right) \\
& =0, \quad\left(\text { since }, f^{\prime}(s)=1-\frac{\lambda(\beta-1)}{n-1} f(s)\right)
\end{aligned}
$$

Thus from above, (79) is satisfied over $D \in\left[\tilde{B}(t), B_{2}\right]$. If $\tilde{B}(t)=0$, we are done. So, now suppose $\tilde{B}(t)>0$.

Consider $D \in[0, \tilde{B}(t))$, then, from (77) we have $r^{*}(D, c, t)=\frac{B_{1}-D}{f\left(T_{1}-t\right)}$ and from (78) we have $J(D, c, t)=$ $Q(c, t)+H(D, c, t)$, where for simplicity of exposition we define $Q(c, t) \triangleq\left(\frac{\left(B_{2}-\tilde{B}(t)\right)^{n}}{c\left(f\left(T_{2}-t\right)\right)^{n-1}}-\frac{\left(B_{1}-\tilde{B}(t)\right)^{n}}{c\left(f\left(T_{1}-t\right)\right)^{n-1}}\right)$ and $H(D, c, t)=\frac{\left(B_{1}-D\right)^{n}}{c\left(f\left(T_{1}-t\right)\right)^{n-1}}$. Substituting in (79) gives,

$$
\begin{aligned}
& L H S=\left(\frac{\partial Q(c, t)}{\partial t}+\lambda\left(E_{z}[Q(Z(c) c, t)]-Q(c, t)\right)\right)+ \\
& \left\{\frac{g\left(r^{*}(D, c, t)\right)}{c}+\frac{\partial H(D, c, t)}{\partial t}+r^{*}(D, c, t) \frac{\partial H(D, c, t)}{\partial D}\right. \\
& \left.+\lambda\left(E_{z}[H(D, Z(c) c, t)]-H(D, c, t)\right)\right\}
\end{aligned}
$$

Using identical steps that lead to (80), it can be shown that the terms within the curly bracket above equal zero. Now consider the first-bracket terms. Let $Q(c, t)=Q_{2}(c, t)-Q_{1}(c, t)$, where $Q_{2}(c, t)=\frac{\left(B_{2}-\tilde{B}(t)\right)^{n}}{c\left(f\left(T_{2}-t\right)\right)^{n-1}}$ and $Q_{1}(c, t)=\frac{\left(B_{1}-\tilde{B}(t)\right)^{n}}{c\left(f\left(T_{1}-t\right)\right)^{n-1}}$. We have,

$$
\begin{aligned}
& \frac{\partial Q_{2}(c, t)}{\partial t}+\lambda\left(E_{z}\left[Q_{2}(Z(c) c, t)\right]-Q_{2}(c, t)\right)= \\
& =\frac{(n-1)\left(B_{2}-\tilde{B}(t)\right)^{n}}{c\left(f\left(T_{2}-t\right)\right)^{n}}\left(-\frac{\tilde{B}^{\prime}(t) f\left(T_{2}-t\right) n}{\left(B_{2}-\tilde{B}(t)\right)(n-1)}+1\right)
\end{aligned}
$$

A similar expression as above is obtained for the term $Q_{1}(c, t)$. Combining the two and using $\frac{B_{1}-\tilde{B}(t)}{f\left(T_{1}-t\right)}=\frac{B_{2}-\tilde{B}(t)}{f\left(T_{2}-t\right)}$, gives,

$$
\frac{\partial Q(c, t)}{\partial t}+\lambda\left(E_{z}[Q(Z(c) c, t)]-Q(c, t)\right)=0
$$

This completes the verification that the functions in (77) and (78) satisfy the PDE equation in (79). We now complete the optimality proof by considering a sequence of relaxed problems and taking the appropriate limit as outlined next.

Step 2 - Verification of Optimality: To verify optimality, we view the problem in two stages - first, over the state space $(D, c, t) \in\left[0, B_{2}\right] \times \mathcal{C} \times\left[0, T_{1}\right)$ (transmission over timeperiod $\left.\left[0, T_{1}\right]\right)$ and second over the state space $(D, c, t) \in$ $\left[B_{1}, B_{2}\right] \times \mathcal{C} \times\left[T_{1}, T_{2}\right)$ (transmission over time-period $\left.\left[T_{1}, T_{2}\right]\right)$. As mentioned in Step 1 of the proof, over the state space $(D, c, t) \in\left[B_{1}, B_{2}\right] \times \mathcal{C} \times\left[T_{1}, T_{2}\right)$, the problem is identical to the $B T$-problem, where $\left(B_{2}-D\right)$ bits remain in the buffer and these need to be transmitted in time $\left(T_{2}-t\right)$. The rate function in (31) reduces to $r^{*}(D, c, t)=\frac{B_{2}-D}{f\left(T_{2}-t\right)}$ and this has been shown to be the optimal policy; see Appendices A and B. Thus, the optimality of $r^{*}(D, c, t)$ and $J(D, c, t)$ over the second stage follows directly from that of the $B T$-problem.

Now consider the first stage, i.e. the state space $(D, c, t) \in$ $\left[0, B_{2}\right] \times \mathcal{C} \times\left[0, T_{1}\right)$. This stage corresponds to transmission over time-period $\left[0, T_{1}\right]$. Once we reach time $t=T_{1}$, we know from the preceding paragraph the optimal policy to be followed thereafter in the second stage. Thus, for the optimization over the first stage, we can abstract the second stage energy cost as a terminal cost incurred at time $T_{1}$ given the particular terminal state. Specifically, the terminal cost function is given as, $h(D, c)=\frac{\left(B_{2}-D\right)^{n}}{c\left(f\left(T_{2}-T_{1}\right)\right)^{n-1}}, D \in\left[B_{1}, B_{2}\right]$ (since this is the minimum (expected) energy cost required to transmit the remaining $\left(B_{2}-D\right)$ bits by time $\left(T_{2}-T_{1}\right)$ ), 
and $h(D, c)=\infty, D \in\left[0, B_{1}\right)$ (since there is a deadline constraint of $T_{1}$ for the first $B_{1}$ bits, and an infinite penalty cost is incurred if $\left.D<B_{1}\right)$. Since this is a non-continuous terminal cost function, we cannot directly apply standard verification results to show optimality. To circumvent this problem, we consider a sequence of relaxed problems $\left\{\mathcal{P}_{k}\right\}$, where the hard deadline constraint on the $B_{1}$ bits is relaxed and instead a sequence of smooth terminal cost functions is assigned, which monotonically converge to the required function above. This is analogous to the steps followed earlier for the $B T$-problem and they are outlined below.

Consider a sequence of numbers $\left\{\tau_{k}\right\}_{k=1}^{\infty}$, where $\tau_{k}>0$ and $\tau_{k} \downarrow 0$. Define a sequence of functions $\left\{f^{k}(s)\right\}_{k=1}^{\infty}$, where each $f^{k}(s)$ satisfies the ODE, $\left(f^{k}\right)^{\prime}(s)=1-\frac{\lambda(\bar{\beta}-1)}{n-1} f^{k}(s)$ with the initial condition $f^{k}(0)=\tau_{k}$. Thus,

$$
\begin{aligned}
f^{k}(s)=\frac{(n-1)}{\lambda(\beta-1)} & \left(1-\exp \left(-\frac{\lambda(\beta-1) s}{n-1}\right)\right) \\
& +\tau_{k} \exp \left(-\frac{\lambda(\beta-1) s}{n-1}\right), s \geq 0
\end{aligned}
$$

Consider now a sequence of relaxed problems, $\left\{\mathcal{P}_{k}\right\}$, over the state space $(D, c, t) \in\left[0, B_{2}\right] \times \mathcal{C} \times\left[0, T_{1}\right)$. Each problem $\mathcal{P}_{k}$ is identical to the $B T$-problem in terms of the system dynamics except that at time $T_{1}$, instead of the hard deadline, a terminal cost is assigned. This terminal cost function is denoted as $h^{k}(D, c)$ and is taken as follows,

$h^{k}(D, c)=\left\{\begin{array}{l}\frac{\left(B_{2}-D\right)^{n}}{c\left(f^{k}\left(T_{2}-T_{1}\right)\right)^{n-1}}, \quad \tilde{B}^{k}\left(T_{1}\right) \leq D<B_{2} \\ \frac{\left(B_{1}-D\right)^{n}}{c\left(f^{k}(0)\right)^{n-1}}+\frac{\left(B_{2}-\tilde{B}^{k}\left(T_{1}\right)\right)^{n}}{c\left(f^{k}\left(T_{2}-T_{1}\right)\right)^{n-1}}-\frac{\left(B_{1}-\tilde{B}^{k}\left(T_{1}\right)\right)^{n}}{c\left(f^{k}(0)\right)^{n-1}} \\ 0 \leq D<\tilde{B}^{k}\left(T_{1}\right)\end{array}\right.$

where in the above equation, the function $\tilde{B}^{k}(t), t \in\left[0, T_{1}\right]$ for the relaxed problem $\mathcal{P}_{k}$ is correspondingly defined as,

$$
\tilde{B}^{k}(t)= \begin{cases}0, & \text { if } \frac{B_{1}}{f^{k}\left(T_{1}-t\right)}<\frac{B_{2}}{f^{k}\left(T_{2}-t\right)} \\ \frac{B_{1}}{\frac{f^{k}\left(T_{1}-t\right)}{1}-\frac{B_{2}}{f^{k}\left(T_{2}-t\right)}}, \frac{1}{f^{k}\left(T_{2}-t\right)}, & \text { otherwise }\end{cases}
$$

Note that since $f^{k}(0)=\tau_{k}$, as we consider larger values of $k$ then $\tau_{k}$ goes to zero and $\tilde{B}^{k}\left(T_{1}\right)$ converges to $B_{1}$ while $f^{k}(s)$ converges to $f(s)$. Thus, we see that the terminal cost function $h^{k}(D, c)$ converges to the desired function as mentioned earlier.

For the relaxed problem $\mathcal{P}_{k}$ the system operates as follows. Given a transmission policy, denoted as $r_{k}(D, c, t)$, the system starts with $D(0)=0$. As this policy is followed, at time $T_{1}$, the terminal cost $h^{k}\left(D\left(T_{1}\right), c\left(T_{1}\right)\right)$ is incurred and the system stops. Also, during the period $t \in\left[0, T_{1}\right]$, if $D(t)=B_{2}$ then all the data has been transmitted and there is no terminal cost incurred. Thus, we see that the relaxed problem $\mathcal{P}_{k}$ is a wellposed, continuous-time control problem with smooth terminal cost functions. Consider now the following rate function,

$$
r_{k}^{*}(D, c, t)=\max _{j:\left(B_{j} \geq D, T_{j} \geq t\right)} \frac{B_{j}-D}{f^{k}\left(T_{j}-t\right)}
$$

and the following minimum cost function which is denoted as $J^{k}(D, c, t)$,

$$
J^{k}(D, c, t)=\left\{\begin{array}{l}
\frac{\left(B_{2}-D\right)^{n}}{c\left(f^{k}\left(T_{2}-t\right)\right)^{n-1}}, \quad \tilde{B}^{k}(t) \leq D \leq B_{2} \\
\frac{\left(B_{1}-D\right)^{n}}{c\left(f^{k}\left(T_{1}-t\right)\right)^{n-1}}+\frac{\left(B_{2}-\tilde{B}^{k}(t)\right)^{n}}{c\left(f^{k}\left(T_{2}-t\right)\right)^{n-1}} \\
-\frac{\left(B_{1}-\tilde{B}^{k}(t)\right)^{n}}{c\left(f^{k}\left(T_{1}-t\right)\right)^{n-1}}, 0 \leq D<\tilde{B}^{k}(t)
\end{array}\right.
$$

Following an identical set of arguments as done in the first step of this proof, it can be seen that the above functions satisfy the HJB equation (note that the functional forms are analogous to those earlier except with $f^{k}(s)$ replacing $f(s)$ and $\tilde{B}^{k}(t)$ replacing $\tilde{B}(t))$. It is also easy to see that the minimum cost function also satisfies the boundary conditions, i.e. it equals the terminal cost function $h^{k}(D, c)$ and also equals zero for $D=B_{2}$. Using the standard verification result, outlined earlier in Lemma 1, it can be seen that the rate function in (82) gives the optimal transmission policy for the relaxed problem $\mathcal{P}_{k}$.

Now consider the limit $k \rightarrow \infty$, then, $J^{k}(D, c, t)$ converges to $J(D, c, t)$ (given in (78)) and $r_{k}^{*}(D, c, t)$ converges to $r^{*}(D, c, t)$. Utilizing the result of Theorem $\mathrm{V}$ (an analogous version as stated below), the optimality of $J(D, c, t)$ and $r^{*}(D, c, t)$ for the first stage of the two-packet problem follows.

Theorem VI: (Two Packet Case): Let $(D, c, t) \in\left[0, B_{2}\right] \times$ $\mathcal{C} \times\left[0, T_{1}\right)$ and define $J(D, c, t) \triangleq \lim _{k \rightarrow \infty} J^{k}(D, c, t)$. Let $J(D, c, t)$ satisfy the HJB equation in (28) and let $r^{*}(D, c, t)$ be an admissible policy for the first stage of the two-packet problem, such that $r^{*}$ is the minimizing value of $r$ in (28). Then,

1) $J(D, c, t) \leq J_{r}(D, c, t), \quad \forall r(\cdot)$ admissible (where $J_{r}(D, c, t)$ denotes the cost-to-go function for that policy)

2) $r^{*}(D, c, t)$ is the optimal policy and $J(D, c, t)$ is the minimum cost function

Proof: The proof is identical to that of Theorem V.

The requirements of the above verification theorem are satisfied. First, from Step 1 we know that $J(D, c, t)$ and $r^{*}(D, c, t)$ satisfy the HJB equation. The function $J(D, c, t)$ also satisfies the boundary condition, i.e. $J\left(D, c, T_{1}\right)=h(D, c), D \in$ $\left[B_{1}, B_{2}\right]$ (where $h(D, c)$ gives the optimal cost for the second stage). The rate function $r^{*}(D, c, t)$ is non-negative and the deadline constraint of $T_{1}$ for the $B_{1}$ bits is also satisfied. This is because from the $B T$-problem we know that $\frac{B_{1}-D}{f\left(T_{1}-t\right)}$ is an admissible rate function that meets the required deadline constraint. Here, since $r^{*}(D, c, t)$ is chosen as the maximum among $\frac{B_{1}-D}{f\left(T_{1}-t\right)}$ and $\frac{B_{2}-D}{f\left(T_{2}-t\right)}$, the transmission rate selected ensures that at least $B_{1}$ bits have been transmitted by time $T_{1}$ (almost surely). 


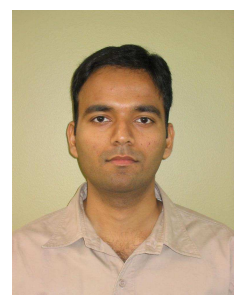

Murtaza Zafer received his B.Tech degree in Electrical Engineering from the Indian Institute of Technology (IIT) Madras, India, in 2001, his S.M. and $\mathrm{Ph} . \mathrm{D}$. degrees in Electrical Engineering and Computer Science from the Massachusetts Institute of Technology (MIT), MA, USA, in 2003 and 2007 respectively. Currently, he is a Research Staff Member at IBM Thomas J. Watson Research Center, NY, USA, where his research is on communication and sensor networks. He spent the summer of 2004 at the Mathematical Sciences Research center, Bell

Laboratories Alcatel-Lucent Inc., and the summer of 2003 at Qualcomm, Inc.

His research interests are in queueing theory, information theory, control and optimization theory, algorithms and protocol design with applications to communication networks. He is the co-recipient of the best Student Paper award at WiOpt conference, 2005. He also received the Siemens and Philips award for academic excellence in 2001.

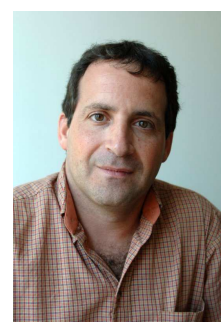

Eytan Modiano received his B.S. degree in Electrical Engineering and Computer Science from the University of Connecticut at Storrs in 1986 and his M.S. and $\mathrm{PhD}$ degrees, both in Electrical Engineering, from the University of Maryland, College Park, MD, in 1989 and 1992 respectively. He was a Naval Research Laboratory Fellow between 1987 and 1992 and a National Research Council Post Doctoral Fellow during 1992-1993. Between 1993 and 1999 he was with MIT Lincoln Laboratory where he was the project leader for MIT Lincoln Laboratory's Next Generation Internet (NGI) project. Since 1999 he has been on the faculty at MIT; where he is presently an Associate Professor. His research is on communication networks and protocols with emphasis on satellite, wireless, and optical networks.

He is currently an Associate Editor for IEEE Transactions on Information Theory, The International Journal of Satellite Communications, and for IEEE/ACM Transactions on Networking. He had served as a guest editor for IEEE JSAC special issue on WDM network architectures; the Computer Networks Journal special issue on Broadband Internet Access; the Journal of Communications and Networks special issue on Wireless Ad-Hoc Networks; and for IEEE Journal of Lightwave Technology special issue on Optical Networks. He was the Technical Program co-chair for Wiopt 2006, IEEE Infocom 2007, and ACM MobiHoc 2007. 